\title{
Utilizing Small Telescopes Operated by Citizen Scientists for Transiting Exoplanet Follow-up
}

Robert T. Zellem ${ }^{1}$ (1), Kyle A. Pearson ${ }^{1,2}$ (1) , Ethan Blaser ${ }^{1,3}$, Martin Fowler ${ }^{4}$, David R. Ciardi ${ }^{5}$ (10, Anya Biferno ${ }^{1}$, Bob Massey ${ }^{6}$, Franck Marchis ${ }^{7,8}{ }^{1}$, Robert Baer ${ }^{9,10}$, Conley Ball ${ }^{11}$, Mike Chasin $^{12,13}$, Mike Conley ${ }^{10}$, Scott Dixon ${ }^{12,13}$, Elizabeth Fletcher ${ }^{14}$, Saneyda Hernandez ${ }^{14}$, Sujay Nair ${ }^{15}$, Quinn Perian ${ }^{15}$, Frank Sienkiewicz ${ }^{16}$, Kalée Tock ${ }^{15}$, Vivek Vijayakumar ${ }^{12}$, Mark R. Swain ${ }^{1}$ (i), Gael M. Roudier ${ }^{1}$ (iD, Geoffrey Bryden ${ }^{1}$, Dennis M. Conti ${ }^{6}$, Dolores H. Hill ${ }^{2}$, Carl W. Hergenrother ${ }^{2}$, Mary Dussault ${ }^{16}$ (1), Stephen R. Kane ${ }^{17}$ (1), Michael Fitzgerald ${ }^{18}$, Pat Boyce $^{12}$, Laura Peticolas ${ }^{19}$, Wilfred Gee ${ }^{20}$ (1), Lynn Cominsky ${ }^{19}$ (1), Rachel Zimmerman-Brachman ${ }^{1}$, Denise Smith ${ }^{21}$ (D) Michelle J. Creech-Eakman ${ }^{22}$, John Engelke ${ }^{1,23}$, Alexandra Iturralde ${ }^{1,24}$,

Diana Dragomir ${ }^{25,26,29}$ (1) , Nemanja Jovanovic ${ }^{27}$, Brandon Lawton ${ }^{21}$ (i), Emmanuel Arbouch ${ }^{8}$, Marc Kuchner ${ }^{28}$ (10), and Arnaud Malvache ${ }^{8}$

${ }^{1}$ Jet Propulsion Laboratory, California Institute of Technology, 4800 Oak Grove Drive, Pasadena, CA 91109, USA; rzellem@jpl.nasa.gov

${ }^{2}$ Lunar and Planetary Laboratory, University of Arizona, 1629 E University Blvd, Tucson, AZ 85721, USA

${ }^{3}$ University of Virginia, Charlottesville, VA 22904, USA

${ }^{4}$ Citizen Scientist, Les Rocquettes, Orchard Road, South Wonston, Winchester SO21 3EX, UK

${ }^{5}$ NASA Exoplanet Science Institute/California Institute of Technology, MC 314-6, 1200 E California Blvd, Pasadena, CA 91125, USA

${ }^{6}$ American Association of Variable Star Observers, 49 Bay State Rd, Cambridge, MA 02138, USA

SETI Institute, 189 Bernardo Ave, Suite 200, Mountain View, CA 94043, USA

${ }^{8}$ Unistellar, 19 rue Vacon, F-13001 Marseille, France

${ }^{9}$ Southern Illinois University Carbondale, MC 4401, 1245 Lincoln Dr, Carbondale, IL 62901, USA

${ }^{10}$ Citizen CATE Experiment, USA

${ }^{11}$ Laguna Blanca School, 4125 Paloma Dr, Santa Barbara, CA 93110, USA

${ }^{12}$ Boyce Research Initiatives and Education Foundation, USA

${ }^{13}$ San Diego Astronomy Association, USA

${ }^{14}$ Towson University, 8000 York Rd, Towson, MD 21252, USA

${ }^{15}$ Stanford Online High School, 415 Broadway Academy Hall, Floor 2, 8853, Redwood City, CA 94063, USA

${ }^{16}$ The Center for Astrophysics, Harvard \& Smithsonian, 60 Garden Street, Cambridge, MA 02138, USA

${ }^{17}$ Department of Earth and Planetary Sciences, University of California, Riverside, CA 92521, USA

${ }_{18}^{18}$ Edith Cowan University, 270 Joondalup Drive, Joondalup, WA 6027, Australia

${ }^{19}$ Sonoma State University, 1801 East Cotati Ave, Rohnert Park, CA 94928, USA

${ }^{20}$ Macquarie University, Sydney, New South Wales 2109, Australia

${ }^{21}$ Space Telescope Science Institute, 3700 San Martin Drive, Baltimore, MD 21218, USA

${ }^{22}$ Department of Physics, New Mexico Institute of Mining and Technology, 801 Leroy Place, Socorro, NM 87801, USA

${ }^{23}$ Raytheon Intelligence, Information, and Services, 300 N Lake Ave, Suite 1120, Pasadena, CA 91101, USA

${ }^{24}$ The University of New Mexico, Albuquerque, NM 87131, USA

${ }_{25}$ Massachusetts Institute of Technology, 77 Massachusetts Ave, Cambridge, MA 02139, USA

${ }^{26}$ Department of Physics and Astronomy, University of New Mexico, Albuquerque, NM, USA

${ }^{27}$ California Institute of Technology, 1200 East California Boulevard, Pasadena, CA 91125, USA

${ }^{28}$ NASA Goddard Space Flight Center, 8800 Greenbelt Rd, Greenbelt, MD 20771, USA

Received 2019 September 26; accepted 2020 March 11; published 2020 April 8

\begin{abstract}
Due to the efforts by numerous ground-based surveys and NASA's Kepler and Transiting Exoplanet Survey Satellite (TESS), there will be hundreds, if not thousands, of transiting exoplanets ideal for atmospheric characterization via spectroscopy with large platforms such as James Webb Space Telescope and ARIEL. However their next predicted mid-transit time could become so increasingly uncertain over time that significant overhead would be required to ensure the detection of the entire transit. As a result, follow-up observations to characterize these exoplanetary atmospheres would require less-efficient use of an observatory's time-which is an issue for large platforms where minimizing observing overheads is a necessity. Here we demonstrate the power of citizen scientists operating smaller observatories $(\leqslant 1 \mathrm{~m})$ to keep ephemerides "fresh," defined here as when the $1 \sigma$ uncertainty in the mid-transit time is less than half the transit duration. We advocate for the creation of a community-wide effort to perform ephemeris maintenance on transiting exoplanets by citizen scientists. Such observations can be conducted with even a 6 inch telescope, which has the potential to save up to $\sim 10,000$ days for a 1000-planet survey. Based on a preliminary analysis of 14 transits from a single 6 inch MicroObservatory telescope, we empirically estimate the ability of small telescopes to benefit the community. Observations with a
\end{abstract}

\footnotetext{
${ }^{29}$ NASA Hubble Fellow.
} 
small-telescope network operated by citizen scientists are capable of resolving stellar blends to within 5 "/pixel, can follow-up long period transits in short-baseline TESS fields, monitor epoch-to-epoch stellar variability at a precision $0.67 \% \pm 0.12 \%$ for a $11.3 \mathrm{~V}$-mag star, and search for new planets or constrain the masses of known planets with transit timing variations greater than two minutes.

Key words: techniques: photometric - surveys - ephemerides - planets and satellites: detection

Online material: color figures

\section{Introduction}

NASA's Transiting Exoplanet Survey Satellite (TESS; Ricker et al. 2014) is predicted to discover 10,000+ transiting exoplanets in its full-frame images (Barclay et al. 2018), crucially providing hundreds of bright targets with large scale heights that are ideal for detailed follow-up spectroscopic characterization by Hubble, the James Webb Space Telescope (JWST), ARIEL, and other next-generation platforms (Zellem et al. 2017, 2019b; Kempton et al. 2018). However, TESS will sample some of its targets for only 27 days and, even in the continuous viewing zone, TESSs nominal mission will end before the launch of JWST, ARIEL, and an Astro2020 Decadal Mission (2021, 2028, and 2030, respectively). As a result, the ephemerides of many of these targets and all previously discovered transiting exoplanets could become "stale," i.e., where the $1 \sigma$ uncertainty in the mid-transit time $\Delta T_{\text {mid }}$ exceeds half the transit duration $t_{\text {dur }}$ (assuming an observing strategy where the planet is observed the same amount of time in-transit as out-of-transit; Figure 1). Since this uncertainty increases with time, a significant amount of observing time must be invested to recover the complete transit. For example, a planet with an uncertainty of just 1 minute in both its orbital period and mid-transit time will have an uncertainty of $\sim 15 \mathrm{hr}$ in its mid-transit time in $10 \mathrm{yr}$ (see Equation (3); see also Dragomir et al. 2019a).

In some cases, the transit duration can be relatively long and observed from the ground provided that the uncertainty in the mid-transit time is much less than the transit duration. Even observing just the ingress or egress is still extremely valuable for bounding the solution space. Therefore even partial observations of long transit durations can still serve to help refine a transit's ephemeris, as has been done for the hot Jupiter HD 80606b (Fossey et al. 2009; Garcia-Melendo \& McCullough 2009).

Given that JWST will study tens to 100-200 transiting exoplanets in detail (Cowan et al. 2015; Kempton et al. 2018) and that ARIEL will survey $\sim 1000$ transiting exoplanets (Tinetti et al. 2016, 2018; Edwards et al. 2019; Zellem et al. 2019b), ephemerides maintenance is required to keep predicted transit times "fresh" $\left(\Delta T_{\text {mid }} \leqslant 0.5 t_{\text {dur }}\right)$. Combating this problem requires follow-up observations from either ground- (e.g., Hellier et al. 2019; Mallonn et al. 2019) or space-based platforms (e.g., with Spitzer; Benneke et al. 2017) to reduce the uncertainties on a planets orbital period and mid-transit time. Here we examine how the transit uncertainties increase as a function of time for the current TESS Objects of Interest (TOIs), confirmed TESS targets, and currently known transiting exoplanets and illustrate the power of a network of small telescopes $(\leqslant 1 \mathrm{~m})$ operated by citizen scientists to keep ephemerides fresh, provide comparatively higher spatial resolution, help confirm long-period planets, monitor epochto-epoch stellar variability, and leverage transit timing variations (TTVs) to measure planetary masses or discover new planets.

\section{Transit Maintenance is Critical for Atmospheric Characterization}

\subsection{Analytic Derivation of Mid-transit Uncertainty}

The time of an exoplanet's next transit or eclipse can be calculated via:

$$
T_{\text {mid }}=n_{\text {orbit }} \cdot P+T_{0}
$$

where $T_{\text {mid }}$ is the time of an upcoming transit $n$ exoplanetary orbits $n_{\text {orbit }}$ in the future, $P$ is the orbital period of the planet, and $T_{0}$ is the planet's mid-transit or mid-eclipse time. Performing error propagation on this equation, one finds:

$$
\begin{aligned}
\operatorname{var}\left(T_{\text {mid }}\right)= & n_{\text {orbit }}^{2} \cdot \operatorname{var}(P) \\
& +2 n_{\text {orbit }} \cdot \operatorname{cov}\left(P, T_{0}\right) \\
& +\operatorname{var}\left(T_{0}\right)
\end{aligned}
$$

and, after linearization:

$$
\begin{aligned}
\Delta T_{\text {mid }}= & \left(n_{\text {orbit }}^{2} \cdot \Delta P^{2}\right. \\
& +2 n_{\text {orbit }} \cdot \Delta P \Delta T_{0} \\
& \left.+\Delta T_{0}^{2}\right)^{1 / 2} .
\end{aligned}
$$

Due to the $n_{\text {orbit }}$ term, the uncertainty in measuring the next transit $\Delta T_{\text {mid }}$ is mostly dependent upon the uncertainty in the orbital period $\Delta P$ compared to the uncertainty in the transit ephemeris $\Delta T_{0}$. In addition, short-period planets run the risk of becoming stale faster than long-period planets with similar uncertainties in their orbital period $\Delta P$ and mid-transit time $\Delta T_{0}$, as the number of elapsed orbits $n_{\text {orbit }}$ in a given amount of time is inversely proportional to the orbital period. Therefore, if an exoplanet has a sufficiently large uncertainty in its midtransit time or orbital period, then it will have a large uncertainty in its next expected mid-transit or mid-eclipse time. A similar expression has been independently derived by 


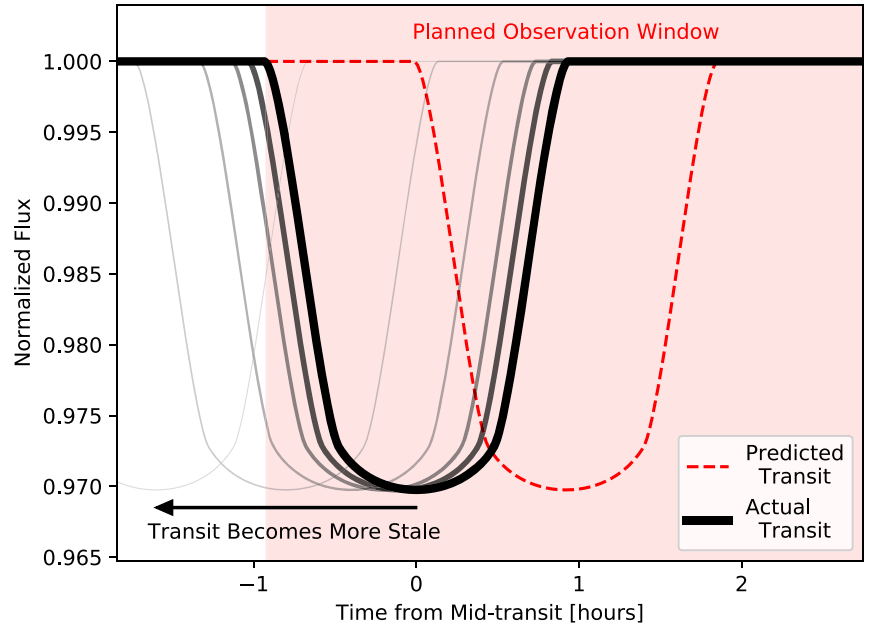

Figure 1. If the uncertainty in the mid-transit time is greater than or equal to half of the transit duration, then one runs the risk of not observing any pretransit baseline (thereby making the identification and removal of systematic errors more difficult) or missing part or all of the transit. In the figure above, an astronomer has planned an observing run (red shaded region) based on a predicted transit time (dashed red line). However, due to uncertainties in the planet's period and mid-transit time, the midpoint of the upcoming transit actually occurs half a transit duration earlier than anticipated (solid black line), resulting in the lack of pre-transit baseline.

(A color version of this figure is available in the online journal.)

Kane et al. (2009), Dragomir et al. (2019a), and Mallonn et al. (2019).

Assuming an observing strategy where an equal amount of time is spent integrating both in- and out-of-transit, then we define here that a transiting exoplanet's ephemeris will become "stale" when the $1 \sigma$ mid-transit uncertainty $\Delta T_{\text {mid }}$ exceeds half the transit duration $t_{\text {dur. }}{ }^{30}$ Such a scenario would run the risk of either partially or completely missing the transit (Figure 1). We can further expand Equation (3):

$$
\begin{aligned}
\Delta T_{\text {mid }}= & \left(n_{\text {orbit }}^{2} \cdot \Delta P^{2}\right. \\
& +2 n_{\text {orbit }} \cdot \Delta P \Delta T_{0} \\
& \left.+\Delta T_{0}^{2}\right)^{1 / 2} \\
& \geqslant 0.5 t_{\text {dur }}
\end{aligned}
$$

and thus derive a figure of merit (FOM) that allows one to quickly determine when a particular transit runs the risk of becoming stale, requiring follow-up observations to reduce the uncertainties in the orbital period $\Delta P$ and mid-transit time $\Delta T_{0}$.

One can also implement the figure of merit that ranks targets ideal for detailed spectral characterization, as independently

\footnotetext{
30 Note that we base our definition on the $1 \sigma$ mid-transit uncertainty. However, if a publication has underestimated their $1 \sigma$ uncertainties, then this definition will falsely define a transiting exoplanet as fresh rather than stale; thus one could more conservatively adopt the $3 \sigma$ mid-transit uncertainties to identify stale targets instead. We proceed with the $1 \sigma$ mid-transit uncertainty definition for the rest of this study.
}

derived by Cowan et al. (2015), Zellem et al. (2017), Goyal et al. (2018), Kempton et al. (2018), and Morgan et al. (2019):

$$
\mathrm{FOM}_{\text {transit }}=\frac{2 H_{s} R_{p} R_{s}^{-2}}{10^{0.2 H-\mathrm{mag}}} .
$$

where $H_{s}$ is the planet's scale height, $R_{p}$ and $R_{s}$ are the planet and host star's radii, respectively, and $H$-mag is the host star's $H$-band magnitude. FOM $_{\text {transit }}$ prioritizes planets with large atmospheric spectral modulation and bright host stars and are most likely to be followed-up for detailed atmospheric spectral characterization with future missions; since these targets typically have larger radii $R_{p}$ and brighter host stars, they are also ideal targets for smaller telescopes. By adding in a term that also prioritizes targets that are stale, where $\Delta T_{\text {mid }} \geqslant$ $0.5 t_{\text {dur }}$, we define:

$$
\mathrm{FOM}_{\text {maint }}=\frac{2 H_{s} R_{p} R_{s}^{-2} \cdot \Delta T_{\text {mid }}\left(0.5 t_{\text {dur }}\right)^{-1}}{10^{0.2 H-\text { mag }}} .
$$

$\mathrm{FOM}_{\text {maint }}$ prioritizes not only larger planets with large scale heights around bright host stars (and are therefore conducive to atmospheric characterization) but also those that have the greatest risk of becoming stale, thus requiring ephemeris maintenance. By using Equation (3) to derive $\Delta T_{\text {mid }}$, then this figure of merit also takes into account a planet's uncertainty in its ephemeris $\Delta T_{0}$ and orbital period $\Delta P$.

\subsection{Current Ephemerides Uncertainties}

From the $\sim 4000$ confirmed transiting exoplanets listed on the NASA Exoplanet Archive (Akeson et al. 2013), we rank the top 20 planets requiring ephemeris maintenance at three different epochs (JWST's launch, ARIEL's launch, and an Astro2020 Decadal mission's launch) with $\mathrm{FOM}_{\text {maint }}$ (Equation (6)) in Tables 1-3. Also included are each planet's "observational likelihood" as calculated by $\mathrm{FOM}_{\text {transit }}$ (Equation (5)), which estimates how likely a particular planet is to be observed for future atmospheric characterization. To provide a probability for a target to be spectroscopically characterized in the future, we rank all of the currently known transiting exoplanets via $\mathrm{FOM}_{\text {transit }}$ and then calculate the percentile rank of each planet so that a planet with an observational likelihood of $100 \%$ is the top-ranked planet (and most likely to be observed in the future) while one with a likelihood of $0 \%$ is the "worst-ranked" planet.

As indiciated in Tables 1-3, there are planets that have high probability to be observed with future platforms (as indicated by their high observational likelihood), yet they are at high risk of going stale or will have substantial uncertainties in their mid-transit times. Therefore, ephemeris maintenance of these high-priority targets is necessary to ensure the efficient use of large observatory time. Based on the planet-star area ratio and $V$-magnitude of the host star, a transit detection significance is estimated for a single 6 inch telescope and presented in these tables. However some targets are rather small or the star is dim, 
Table 1

Top 20 Planets with Large Spectral Modulation Also Requiring Ephemeris Maintenance for JWST Launch (2021 March 30)

\begin{tabular}{|c|c|c|c|c|c|c|c|c|c|c|}
\hline Planet Name & $V$-mag & $H$-mag & $\begin{array}{c}\text { Transit } \\
\text { Depth (\%) }\end{array}$ & $\begin{array}{c}\Delta T_{\text {mid }} \\
\text { (minutes) }\end{array}$ & $\begin{array}{c}\text { Transit } \\
\text { Duration } \\
\text { (minutes) }\end{array}$ & $\begin{array}{c}\text { Orbital } \\
\text { Period (days) }\end{array}$ & $\begin{array}{c}\text { Scale } \\
\text { Height } \\
(\mathrm{km})\end{array}$ & $\begin{array}{l}\text { Observational } \\
\text { Likelihood (\%) }\end{array}$ & $\begin{array}{c}\text { Estimated } V \text {-band } \\
\text { Detection Significance } \\
\text { with a } \\
\text { Single } 6 \text { inch } \\
\text { Telescope }(\sigma)\end{array}$ & $\begin{array}{c}\text { Number } \\
\text { of } 6 \text { inch } \\
\text { Telescopes } \\
\text { Needed for a } \\
3 \sigma \text { Detection }\end{array}$ \\
\hline KIC 5951458 b & $\mathrm{N} / \mathrm{A}$ & 11.382 & 0.1558 & 71434382.4 & 2261.69 & 1320.1 & 111.28 & 61.27 & $\mathrm{~N} / \mathrm{A}$ & $\mathrm{N} / \mathrm{A}$ \\
\hline HIP 41378 e & 8.93 & 7.786 & 0.1392 & 1493281.44 & 220.09 & 131.0 & 87.73 & 84.25 & 0.87 & 12 \\
\hline KIC 3558849 b & $\mathrm{N} / \mathrm{A}$ & 12.819 & 0.3891 & 2224569.6 & 1116.53 & 1322.3 & 81.44 & 58.22 & $\mathrm{~N} / \mathrm{A}$ & $\mathrm{N} / \mathrm{A}$ \\
\hline TRAPPIST-1 h & 18.8 & 10.718 & 0.3403 & 507.69 & 83.52 & 18.767 & 78.21 & 93.39 & 0.18 & 275 \\
\hline HD 97658 b & 7.71 & 5.821 & 0.0884 & 678.24 & 183.57 & 9.4909 & 119.27 & 94.57 & 0.75 & 16 \\
\hline Qatar-8 b & 11.526 & 10.005 & 1.0153 & 426.02 & 242.33 & 3.71495 & 888.49 & 98.34 & 3.33 & 1 \\
\hline $\mathrm{K} 2-141 \mathrm{c}$ & 11.389 & 8.524 & 0.8858 & 65.12 & 180.06 & 7.7485 & 1022.57 & 99.9 & 3.01 & 1 \\
\hline TOI 216.02 & 12.324 & 10.332 & 0.6944 & 922.46 & 255.26 & 17.089 & 275.39 & 92.45 & 1.86 & 3 \\
\hline KELT-8 b & 10.833 & 9.269 & 1.2927 & 173.12 & 282.38 & 3.24406 & 911.62 & 99.07 & 5.04 & 1 \\
\hline CoRoT-24 c & $\mathrm{N} / \mathrm{A}$ & 13.046 & 0.2738 & 3521.81 & 315.29 & 11.759 & 169.78 & 72.55 & $\mathrm{~N} / \mathrm{A}$ & $\mathrm{N} / \mathrm{A}$ \\
\hline HD $219134 c$ & 5.57 & 3.469 & 0.0322 & 130.05 & 174.95 & 6.76458 & 78.9 & 95.19 & 0.47 & 42 \\
\hline Kepler-32 f & 16.452 & 12.901 & 0.0228 & 589.59 & 65.77 & 0.74296 & 395.35 & 75.29 & 0.02 & 18915 \\
\hline HAT-P-3 b & 11.577 & 9.542 & 1.2431 & 107.74 & 147.3 & 2.8997 & 217.3 & 94.88 & 4.02 & 1 \\
\hline Kepler-42 d & 16.124 & 11.685 & 0.0865 & 47.61 & 45.08 & 1.865169 & 293.8 & 92.42 & 0.09 & 1116 \\
\hline
\end{tabular}

requiring multiple telescopes to statistically resolve the signal to $\geqslant 3 \sigma$. The number of 6 inch telescopes required to make a $3 \sigma$ detection is also shown in these tables. For a detailed description on how the values for these two columns were calculated, please see Section 4 and Figure 7.

To more easily place the planets discovered by TESS into context with the $\sim 4000$ currently known exoplanets, we construct "representative planets"-planets that adopt median values of the properties of their larger sample — of the currently known exoplanets listed on the NASA Exoplanet Archive, the TESS-discovered exoplanets, and the TOIs. The Representative Known Planet adopts a median orbital period $P=12.06$ days and median uncertainty $\Delta P=8.12 \mathrm{~s}$, a median mid-transit ephemeris $T_{0}=2454985.88$ JD and median uncertainty $\Delta T_{0}=220.28 \mathrm{~s}$, and a median $V$-mag $=13.716$ (Table 4). At the time of this study, thirty-seven TESS-discovered exoplanets have been confirmed (Bakos et al. 2018; Huang et al. 2018; Gandolfi et al. 2018, 2019; Cañas et al. 2019; Dawson et al. 2019; Dragomir et al. 2019b; Dumusque et al. 2019; Esposito et al. 2019; Günther et al. 2019; Huber et al. 2019; Jones et al. 2019; Kipping et al. 2019; Kostov et al. 2019; Luque et al. 2019; Newton et al. 2019; Nielsen et al. 2019; Quinn et al. 2019; Rodriguez et al. 2019; Trifonov et al. 2019; Vanderburg et al. 2019; Vanderspek et al. 2019; Wang et al. 2019; Winters et al. 2019); the Representative TESS Planet adopts their median orbital period $P=5.97$ days and median uncertainty $\Delta P=26.35 \mathrm{~s}$, a median mid-transit ephemeris $T_{0}=2458366.17$ JD and median uncertainty $\Delta T_{0}=64.66 \mathrm{~s}$, and a median $V$-mag $=9.724$. Of the current 1604 TESS Objects of Interest ${ }^{31}$ (TOIs), 1555 have reported mid-transit and orbital period uncertainties; these TOIs have mid-transit and orbital period uncertainties that range from $\sim 0.5 \mathrm{~s}$ to tens of minutes. The Representative TOI Planet adopts their median orbital period $P=3.85$ days and median uncertainty $\Delta P=34.56 \mathrm{~s}$, median mid-transit ephemeris $T_{0}=$ $2458517.96 \mathrm{JD}$ and median uncertainty $\Delta T_{0}=181.87 \mathrm{~s}$, and TESS magnitude of 10.261 .

The TESS Follow-up Observation Program ${ }^{32}$ (TFOP) has done an excellent job to refine the mid-transit and orbital period uncertainties of TOIs in the process of confirming these targets, as evidenced by currently reported high precisions of the confirmed TESS-discovered planets. We conservatively proceed with the TOIs for the rest of the analysis presented here, not only to de-bias our analysis from potential small number statistics (versus the confirmed TESS-discovered planets), but also in case future confirmed TESS-discovered exoplanets do not achieve similarly high timing precisions. Using the currently reported TOI mid-transit and period uncertainties and Equation (3), we estimate how quickly the TOIs would become stale, assuming no additional observations are conducted.

\footnotetext{
31 https://tess.mit.edu/publications/

32 https://heasarc.gsfc.nasa.gov/docs/tess/followup.html
} 
Table 2

Top 20 Planets with Large Spectral Modulation Also Requiring Ephemeris Maintenance for Estimated ARIEL Launch (2028 January 1)

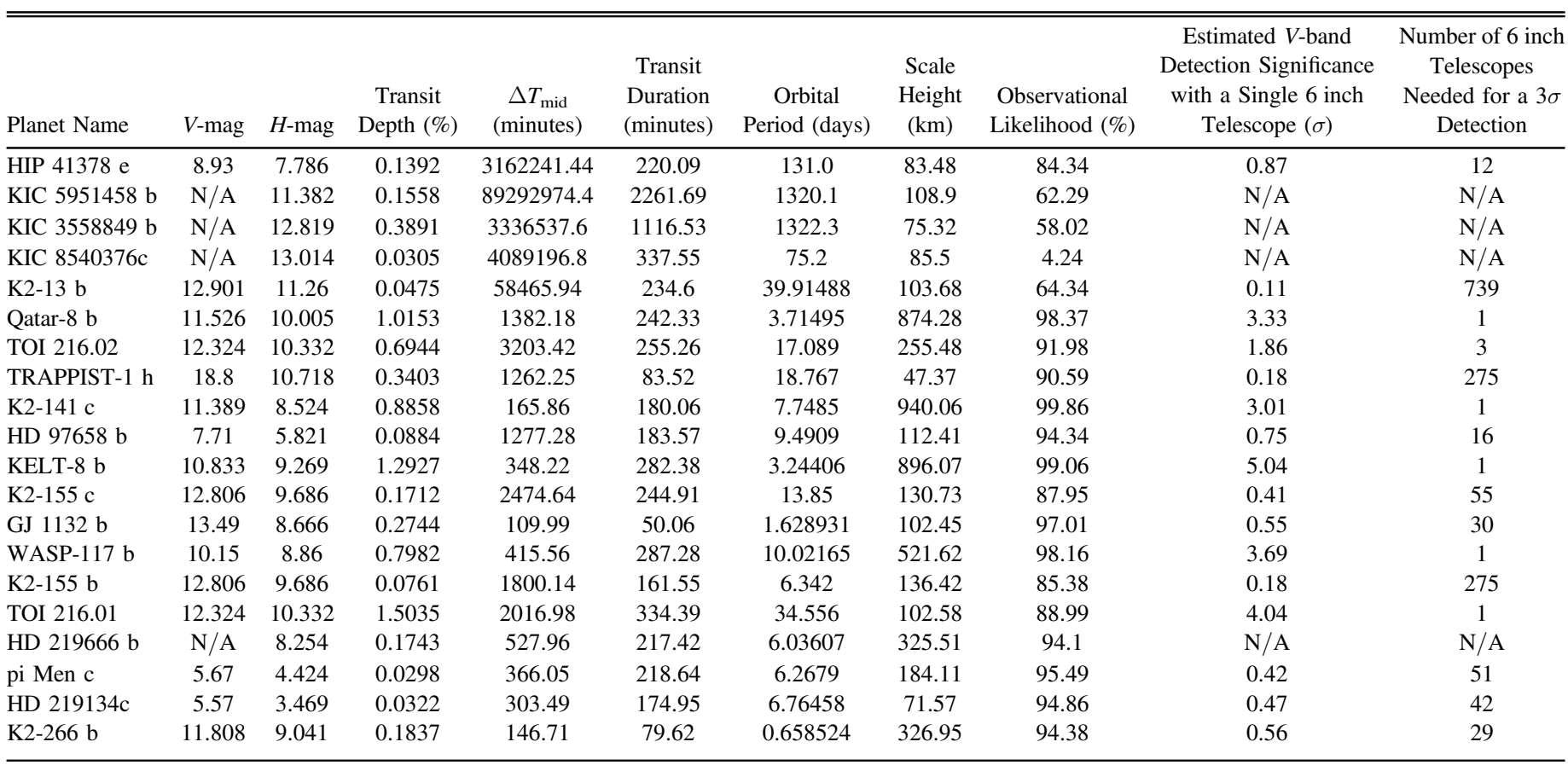

Because the covariance term $\operatorname{cov}\left(P, T_{0}\right)$ is not currently supplied for each TOI, we estimate a global covariance term via:

$$
\operatorname{cov}\left(P, T_{0}\right)=\frac{\sum_{i=1}^{n}\left(\Delta T_{\text {mid }, i}-\overline{\Delta T_{\text {mid }}}\right)\left(\Delta P_{i}-\overline{\Delta P}\right)}{n-1}
$$

where $n$ is the number of TOIs, $\Delta T_{\text {mid, } i}$ is the mid-transit uncertainty of TOI $i, \overline{\Delta T_{\text {mid }}}$ is the mean mid-transit uncertainty of all $n$ TOIs, $\Delta P_{i}$ is the uncertainty of the orbital period of TOI $i$, and $\overline{\Delta P}$ is the mean orbital period uncertainty of all $n$ TOIs. Using this equation, we find $\operatorname{cov}\left(P, T_{0}\right)=0.18 \mathrm{~s}$. We assume that this covariance term is global in that it applies to each individual currently known TOI object. In this manner, we estimate that 698 TOIs ( $45 \%$ total) will become at risk one year after their last observation, 968 TOIs $(62 \%$ total) will become stale in two years, and 1284 TOIs (83\% total) will become stale in five years (Figure 2).

We can similarly calculate how long the timing of the three Representative Planets (Table 4) will remain fresh (Figure 3). We find that the Representative TOI Planet would become stale after $\sim 0.8 \mathrm{yr}$ and have a mid-transit uncertainty of $54.69 \mathrm{~min}-$ utes after $1 \mathrm{yr}$ and $4.55 \mathrm{hr}$ after $5 \mathrm{yr}$, while the Representative Confirmed TESS Planet would become stale after $\sim 3 \mathrm{yr}$, and the Representative Known Planet could remain fresh for over $15 \mathrm{yr}$.
Thus, while a planet may now have a precise estimated upcoming transit time, it can still accrue large uncertainties, especially after many orbits (Equation (3)). Therefore, orbital period and mid-transit maintenance ("ephemerides maintenance") through regular monitoring is necessary to keep transit time uncertainties small so transiting exoplanets can be efficiently followed-up for atmospheric characterization.

A community-wide effort to monitor transiting exoplanets with ground-based telescopes, particularly smaller telescopes $(\leqslant 1 \mathrm{~m})$ used by citizen scientists, could support future missions by alleviating observing overhead. A study by Mallonn et al. (2019), for example, highlights the capability of $0.3 \mathrm{~m}$ through $2.2 \mathrm{~m}$ telescopes to greatly reduce ephemeris uncertainties for 21 hot Jupiters. Similarly, the $200 \mathrm{~mm}$ (7.87-in) WASP-South survey demonstrated the capability of smaller telescopes by recovering the transit of the hot-Neptune HD 219666b ( $V$-mag $=9.9$; transit depth $\left(R_{p} / R_{s}\right)^{2}=0.2 \%$ ); when combined with TESS observations, WASP-South was able to revise the uncertainty in calculating future transit events to 1 minute per year (Hellier et al. 2019).

Here we explore the potential of observations of transiting exoplanets with small telescopes $(\leqslant 1 \mathrm{~m})$ operated by citizen scientists. We ultimately find that, despite their relatively small aperture sizes, these platforms can produce high quality transit observations (Figures 4-6), successfully keep transit times fresh, and enable additional science explored in the following sections. 
Table 3

Top 20 Planets with Large Spectral Modulation Also Requiring Ephemeris Maintenance for Estimated Astro2020 Launch (2030 January 1)

\begin{tabular}{|c|c|c|c|c|c|c|c|c|c|c|}
\hline Planet Name & $V$-mag & $H$-mag & $\begin{array}{c}\text { Transit } \\
\text { Depth (\%) }\end{array}$ & $\begin{array}{c}\Delta T_{\mathrm{mid}} \\
\text { (minutes) }\end{array}$ & $\begin{array}{c}\text { Transit } \\
\text { Duration } \\
\text { (minutes) }\end{array}$ & $\begin{array}{c}\text { Orbital } \\
\text { Period (days) }\end{array}$ & $\begin{array}{l}\text { Scale } \\
\text { Height } \\
(\mathrm{km})\end{array}$ & $\begin{array}{c}\text { Observational } \\
\text { Likelihood (\%) }\end{array}$ & $\begin{array}{l}\text { Estimated } V \text {-band } \\
\text { Detection } \\
\text { Significance with a } \\
\text { Single } 6 \text { inch Tele- } \\
\text { scope }(\sigma)\end{array}$ & $\begin{array}{c}\text { Number of } \\
6 \text { inch } \\
\text { Telescopes } \\
\text { Needed for a } 3 \sigma \\
\text { Detection }\end{array}$ \\
\hline HIP 41378 e & 8.93 & 7.786 & 0.1392 & 3601441.44 & 220.09 & 131.0 & 83.48 & 84.34 & 0.87 & 12 \\
\hline KIC 5951458 b & $\mathrm{N} / \mathrm{A}$ & 11.382 & 0.1558 & 107151566.4 & 2261.69 & 1320.1 & 108.9 & 62.29 & $\mathrm{~N} / \mathrm{A}$ & $\mathrm{N} / \mathrm{A}$ \\
\hline KIC 8540376c & $\mathrm{N} / \mathrm{A}$ & 13.014 & 0.0305 & 4593340.8 & 337.55 & 75.2 & 85.5 & 4.24 & $\mathrm{~N} / \mathrm{A}$ & $\mathrm{N} / \mathrm{A}$ \\
\hline Qatar-8 b & 11.526 & 10.005 & 1.0153 & 1665.86 & 242.33 & 3.71495 & 874.28 & 98.37 & 3.33 & 1 \\
\hline TOI 216.02 & 12.324 & 10.332 & 0.6944 & 3884.54 & 255.26 & 17.089 & 255.48 & 91.98 & 1.86 & 3 \\
\hline TRAPPIST-1 h & 18.8 & 10.718 & 0.3403 & 1486.89 & 83.52 & 18.767 & 47.37 & 90.59 & 0.18 & 275 \\
\hline K2-141 c & 11.389 & 8.524 & 0.8858 & 195.96 & 180.06 & 7.7485 & 940.06 & 99.86 & 3.01 & 1 \\
\hline HD 97658 b & 7.71 & 5.821 & 0.0884 & 1454.69 & 183.57 & 9.4909 & 112.41 & 94.34 & 0.75 & 16 \\
\hline KELT-8 b & 10.833 & 9.269 & 1.2927 & 400.29 & 282.38 & 3.24406 & 896.07 & 99.06 & 5.04 & 1 \\
\hline TOI 216.01 & 12.324 & 10.332 & 1.5035 & 2440.34 & 334.39 & 34.556 & 102.58 & 88.99 & 4.04 & 1 \\
\hline HD 219666 b & $\mathrm{N} / \mathrm{A}$ & 8.254 & 0.1743 & 639.48 & 217.42 & 6.03607 & 325.51 & 94.1 & $\mathrm{~N} / \mathrm{A}$ & $\mathrm{N} / \mathrm{A}$ \\
\hline pi Men c & 5.67 & 4.424 & 0.0298 & 443.55 & 218.64 & 6.2679 & 184.11 & 95.49 & 0.42 & 51 \\
\hline $\mathrm{K} 2-266 \mathrm{~b}$ & 11.808 & 9.041 & 0.1837 & 173.89 & 79.62 & 0.658524 & 326.95 & 94.38 & 0.56 & 29 \\
\hline HD $219134 c$ & 5.57 & 3.469 & 0.0322 & 354.82 & 174.95 & 6.76458 & 71.57 & 94.86 & 0.47 & 42 \\
\hline
\end{tabular}

Table 4

The Representative Planets Adopted for this Study

\begin{tabular}{|c|c|c|c|c|c|}
\hline $\begin{array}{l}\text { Representative } \\
\text { Planet }\end{array}$ & $\begin{array}{l}\text { Host Star } \\
\text { Magnitude }\end{array}$ & $\begin{array}{c}\text { Orbital Period } \\
P \text { (days) }\end{array}$ & $\begin{array}{c}\text { Period Uncertainty } \\
\qquad \Delta P(\mathrm{~s})\end{array}$ & $\begin{array}{l}\text { Mid-transit Time } \\
T_{\text {mid }}(\mathrm{JD})\end{array}$ & $\begin{array}{l}\text { Mid-transit Uncertainty } \\
\qquad \Delta T_{\text {mid }} \text { (s) }\end{array}$ \\
\hline TOI & 10.261 TESS-mag & 3.85 & 34.56 & 2458517.96 & 181.87 \\
\hline TESS & $9.724 V$-mag & 5.97 & 26.35 & 2458366.17 & 64.66 \\
\hline
\end{tabular}

\section{EXOTIC: The EXOplanet Transit Interpretation Code}

To aid the citizen science community, we have developed the EXOplanet Transit Interpretation Code $\left(\right.$ EXOTIC $\left.^{33}\right)$, a complete transit data reduction tool written in Python 3 and aimed at the amateur astronomer. EXOTIC can start with either raw fits files or a pre-reduced timeseries. If raw fits files are used, EXOTIC can calibrate them with flats, darks, and biases, and then perform multi-object optimal aperture photometry. The ideal aperture size, sky annulus (for background count estimation subtraction), and comparison star selection is determined by minimizing the residual scatter in the data after performing a least-squares fit using the scipy.optimize. least_squares package (Virtanen et al. 2019) to the data with a Mandel \& Agol (2002) model transit lightcurve. These

\footnotetext{
$\overline{33 \text { https://github.com/blaserethan/EXOTIC }}$
}

optimal raw photometric data (i.e., the combination of the comparison star, aperture size, and sky annulus that produces the least scatter) are then jointly fit by a model lightcurve and an exponential airmass correction function (e.g., Swain et al. 2010) with a Markov Chain Monte Carlo (MCMC; e.g., Ford 2005). The priors for the MCMC are automatically scraped from the NASA Exoplanet Archive and the limbdarkening parameters are generated from EXOFAST (Eastman et al. 2013); all priors are then confirmed by the user. EXOTIC then calculates the $1 \sigma$ uncertainties on the mid-transit time and transit depth $\left(R_{p} / R_{s}\right)^{2}$ from the MCMC posteriors. EXOTIC can alternatively start with pre-reduced data (time-varying flux) and fit it with a model lightcurve. It also can reduce data in realtime to provide a useful visualization, e.g., for star parties. The EXOTIC reduction code has been fully tested on five different observing platforms and was used to generate all of the data in Figures 4 and 5 as well as the first five lightcurves in Figure 6. 
TOI Mid-Tranist Uncertainty Since Last Observation

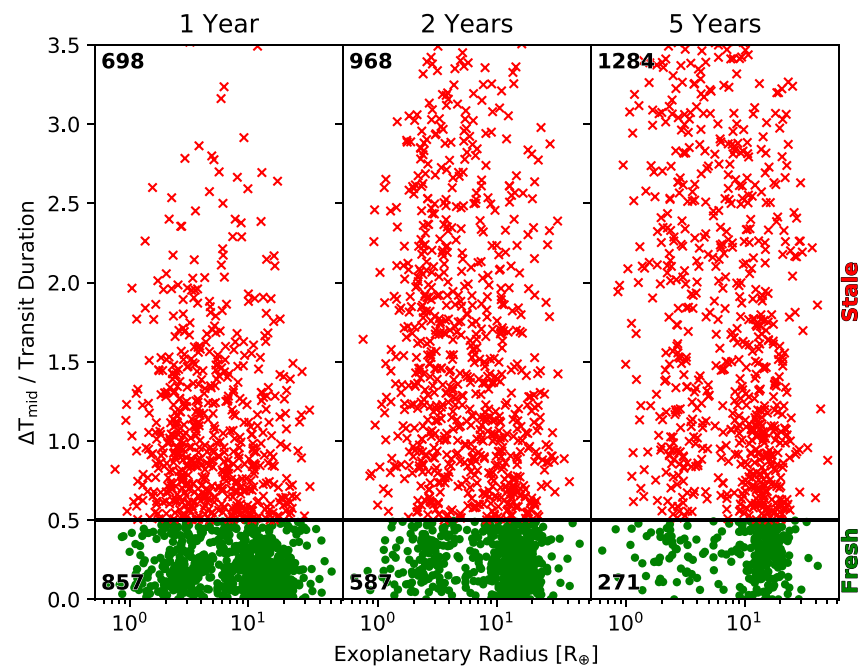

Figure 2. The number of TOIs that transition from fresh (green dots) to stale (red Xs; where the uncertainty in the mid-transit time $\Delta T_{\text {mid }}$ exceeds half the transit duration) as a function of time since their last transit observation. We find that after one year, two years, and five years $45 \%, 62 \%$, and $83 \%$ of the TOI population, respectively, will become stale, requiring ephemerides maintenance.

(A color version of this figure is available in the online journal.)

Using EXOTIC, we find that even a single 6 inch $(15.24 \mathrm{~cm})$ telescope can produce high precision data (Table 5 and Figures 4 and 5). For the relatively dim $12.29 \mathrm{~V}$-mag HAT$\mathrm{P}-32 \mathrm{~b}$, it achieves precisions up to 1.02 minutes for the midtransit time, a $0.05 \%$ transit depth precision $\Delta\left(R_{p} / R_{s}\right)^{2}$ (a $37.58 \sigma$ detection), and a $0.60 \%$ residual rms scatter, despite the target drifting $\sim 300$ pixels on the focal plane over the course of the observation.

\section{Demonstrating and Forecasting the Capability of Small Telescopes}

By analyzing the 14 transit observations by a MicroObservatory telescope (Figures 4 and 5), we forecast the capability of a single 6 inch telescope (adopted here as a typical aperture size operated by a citizen scientist) by empirically deriving the measured residual rms scatter, mid-transit time uncertainty, and transit depth uncertainty $\Delta\left(R_{p} / R_{S}\right)^{2}$ as functions of its host star's $V$-magnitude (Figure 7). These relationships assume that the host star brightness is the largest determiner of the observed precision and that, to first order, these uncertainties scale proportionally to the square root of the number of photons, as indicated in Figure 7. We also conservatively adopt systematic noise floors that are equal to the highest observed precisions $(0.52 \%$ on the rms scatter, $0.05 \%$ on the transit depth $\Delta\left(R_{p} / R_{s}\right)^{2}$, and 1.02 minutes on the mid-transit time). We also conservatively remove these minimum values from our fits as they achieve comparatively much higher precision than the

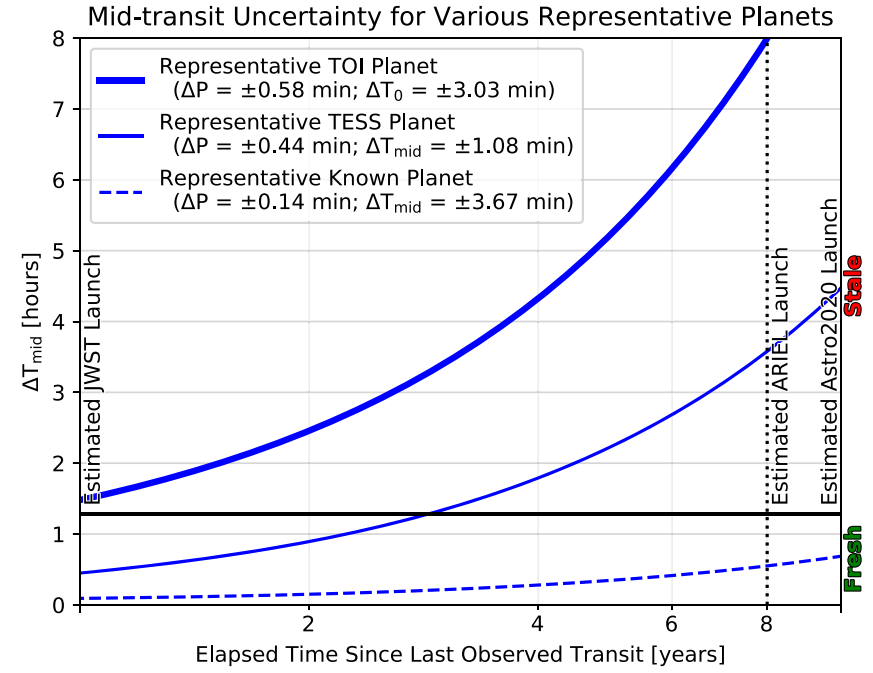

Figure 3. The mid-transit uncertainty as a function of the elapsed time since the last observation of the Representative TESS Planet (blue line) and Known Planet (dashed blue line). The Representative TOI Planet becomes stale on the order of $\sim 3 \mathrm{yr}$, requiring additional observations to keep them fresh for followup observations with large telescopes.

(A color version of this figure is available in the online journal.)

other measurements, in particular the transit depth and midtransit time uncertainties, and might not necessarily reflect typical observations by a single 6 inch telescope. We also find that all three of these uncertainties are not dependent upon the measured planet-to-star radius ratio and that binning in time does not appreciably increase the precision of the MicroObservatory. For example, a single telescope achieves an average precision of $0.83 \%$ per minute for HAT-P-32b (Figure 5); extrapolating this precision over the entire 187.23 minutes transit (Wang et al. 2019b), one would expect it to measure HAT-P-32b's transit depth with a precision of $\Delta\left(R_{p} / R_{s}\right)^{2}=0.06 \%$, however it only achieves a precision of $0.29 \%$. Therefore we conservatively assume that the transit duration is largely negligible in determining the measured transit precision.

Using these empirically derived functions, we estimate that a single 6 inch telescope can measure to $3 \sigma$ the transits of 195 of the currently known transiting exoplanets listed on the NASA Exoplanet Archive (Figure 8). Thus, even now, there are many targets that are accessible with a small telescope. We anticipate that this number will only increase as more planets are discovered by TESS, which is predicted to discover hundreds, if not thousands, of exoplanets that feature large transit depths orbiting bright host stars (Sullivan et al. 2015; Barclay et al. 2018). We also find that of the top 20 currently known targets (Tables 1-3) ranked by our Figure of Merit FOM $_{\text {maint }}$ (Equation (6)), a single 6 inch telescope can measure the transit depth $\left(R_{p} / R_{s}\right)^{2}$ of 5 of the potential JWST targets to $3 \sigma$ (Table 1 ), thereby providing more accurate transit timings for 

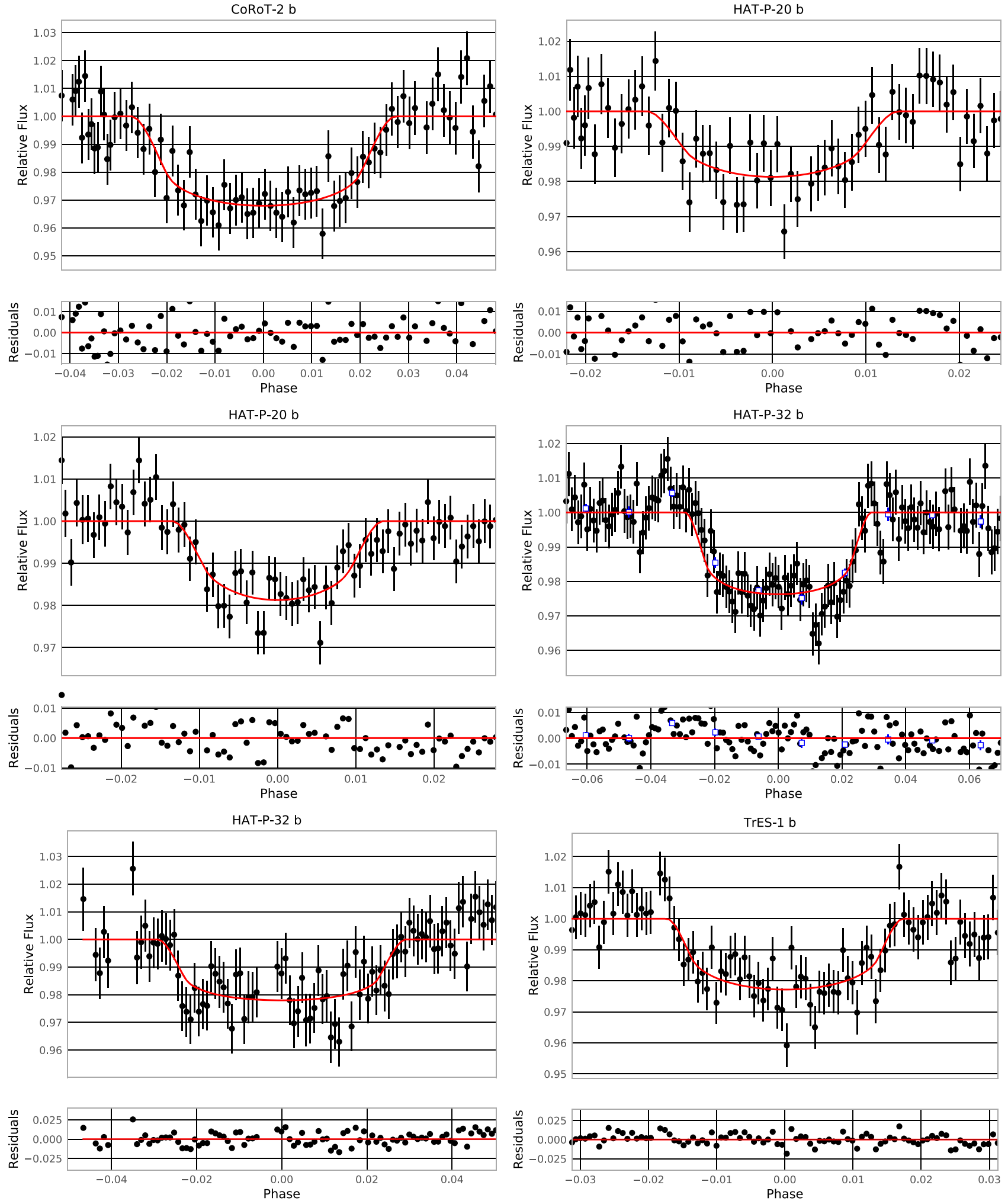

Figure 4. A sample of transits observed with a single 6 inch $(15.24 \mathrm{~cm})$ MicroObservatory telescope (Sadler et al. 2001). Despite most of these targets being relatively dim, the MicroObservatory can still achieve high observational precision (Table 5).

(A color version of this figure is available in the online journal.) 

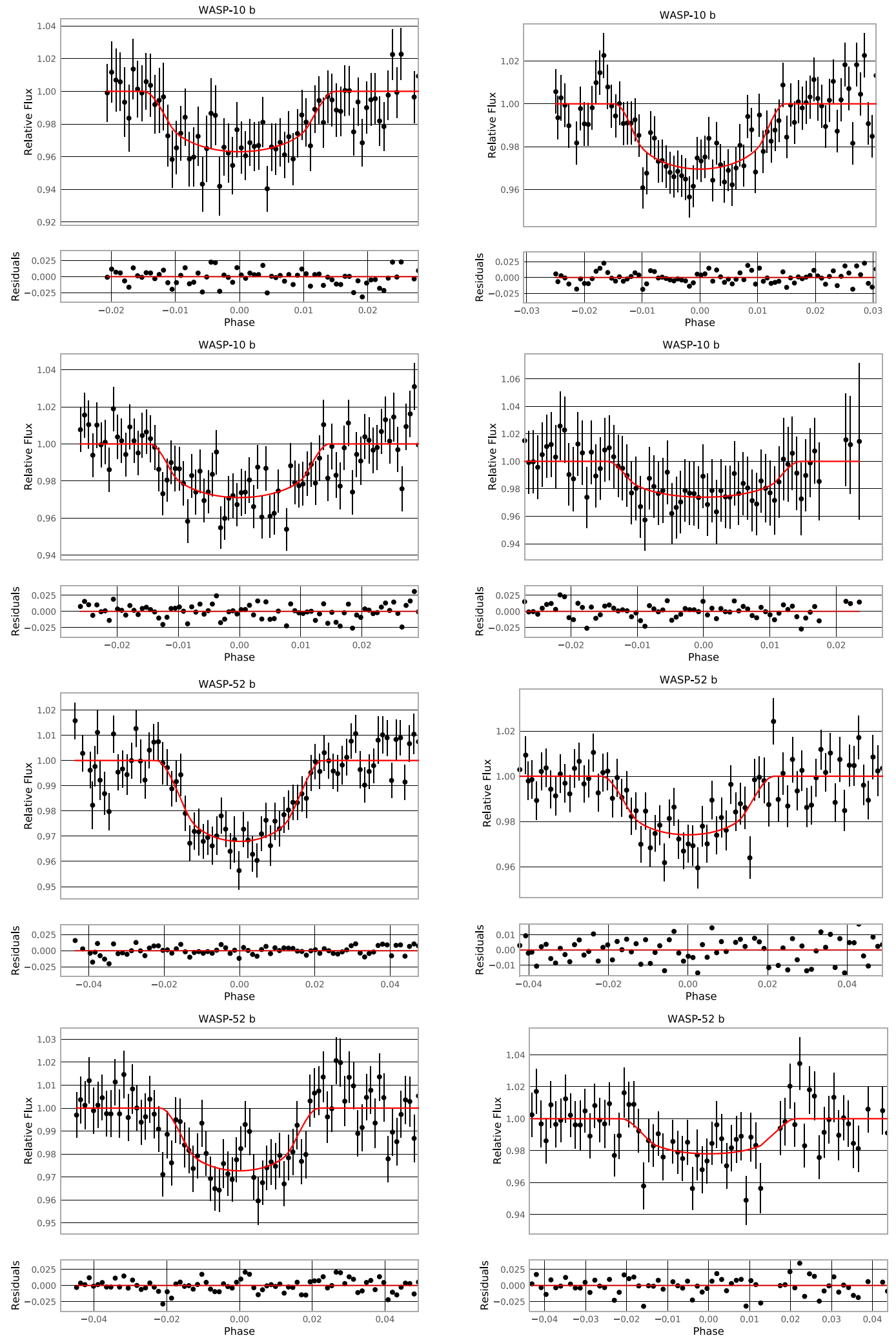

Figure 5. Same as Figure 4.

(A color version of this figure is available in the online journal.) 

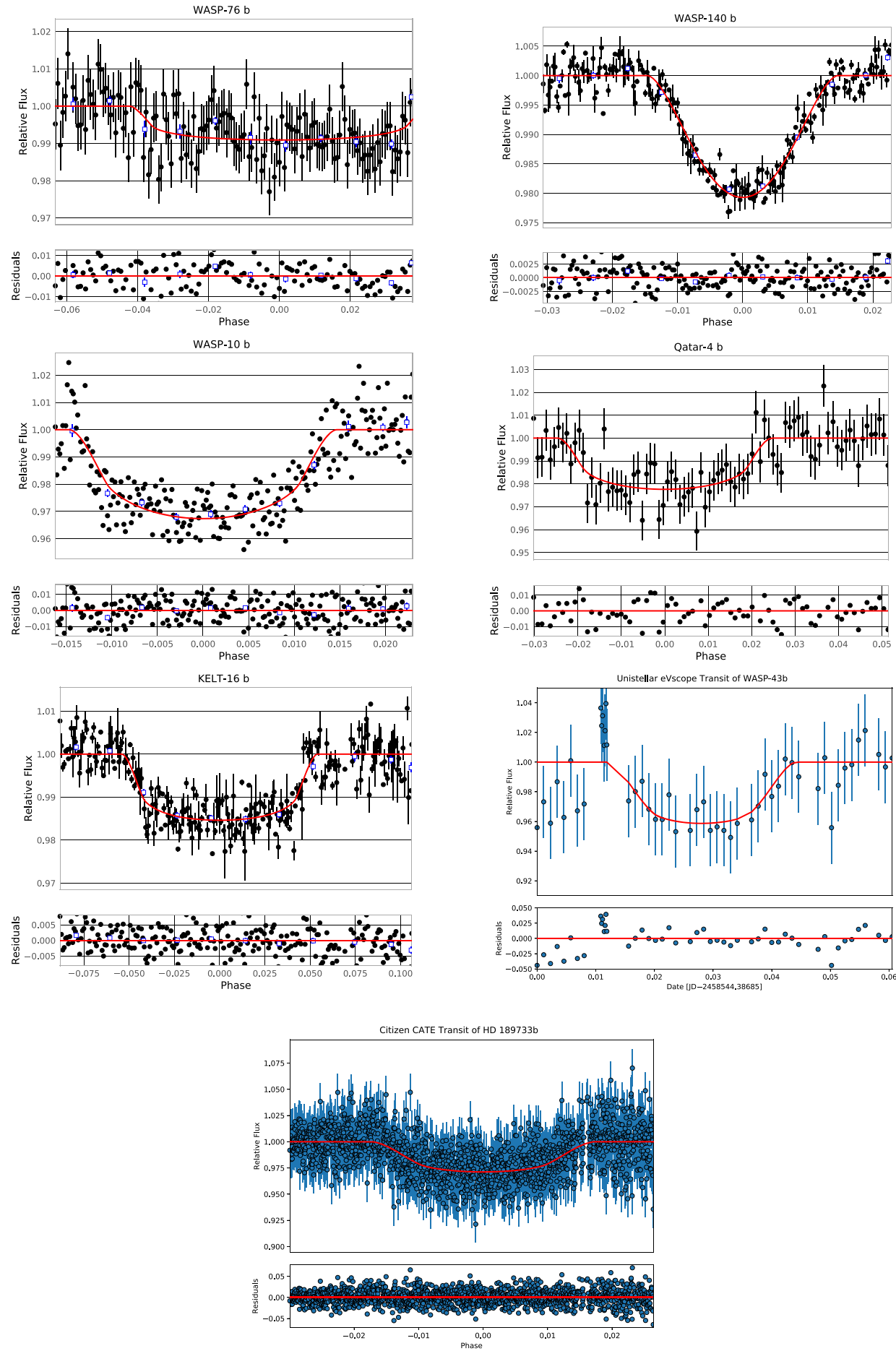

Figure 6. Sample transiting exoplanets observed with small telescopes. First Row: Las Cumbres Observatory $0.4 \mathrm{~m}$ observations of WASP-76b (9.5 V-mag) and WASP-140b (11.1 V-mag). Second Row: WASP-10b (12.7 V-mag; Left) observed with a 16 inch $(40.64 \mathrm{~cm})$ Sierra Remote Observatory robotic telescope and Qatar4b (13.60 V-mag; Right) observed with a 14 inch $(35.56 \mathrm{~cm})$ telescope. Third Row: KELT-16b (11.898 V-mag; Left) observed with an 11 inch $(27.94 \mathrm{~cm})$ telescope and WASP-43b $(V$-mag $=12.4)$ observed with a 4.5 inch $(11.43 \mathrm{~cm})$ Unistellar eVscope (Marchis et al. 2020). Bottom: HD 189733b $(V$-mag $=7.648)$ observed with a 3.15 inch $(8 \mathrm{~cm}$ ) Citizen CATE (Penn et al. 2017, 2019). All the data sets, except WASP-43b, were operated and/or reduced by amateur astronomers and the first five lightcurves were fully reduced with EXOTIC (Section 3). Blue squares are the bins of 10 data points to help aid the eye.

(A color version of this figure is available in the online journal.) 
Table 5

Measured Uncertainties for 14 Transiting Exoplanet Observations with a Single 6 inch (15.24 cm) MicroObservatory Telescope

\begin{tabular}{|c|c|c|c|c|c|c|}
\hline $\begin{array}{l}\text { Planet } \\
\text { Name }\end{array}$ & $\begin{array}{l}\text { Host Star } \\
V \text {-mag }\end{array}$ & $\begin{array}{c}\text { Residual Rms } \\
\text { Scatter }(\% / \text { minute })\end{array}$ & $\begin{array}{c}\text { Mid-transit Time } T_{0} \\
\text { (BJD_TDB-2400000.5) }\end{array}$ & $\begin{array}{c}\text { Mid-transit Time } \\
\text { Uncertainty } \Delta T_{0} \text { (minutes) }\end{array}$ & $\begin{array}{l}\text { Transit Depth } \\
\left(R_{p} / R_{s}\right)^{2}(\%)\end{array}$ & $\begin{array}{c}\text { Transit Depth } \\
\text { Uncertainty } \Delta\left(R_{p} / R_{s}\right)^{2}(\%)\end{array}$ \\
\hline CoRoT-2b & 12.57 & 0.75 & 58746.1678 & 4.27 & 2.59 & 0.35 \\
\hline HAT-P-20b & 11.339 & 0.73 & 58131.1368 & 4.38 & 1.66 & 0.31 \\
\hline HAT-P-20b & 11.339 & 0.52 & 58818.3441 & 5.52 & 1.67 & 0.3 \\
\hline HAT-P-32b & 11.289 & 0.83 & 58739.3143 & 5.5 & 1.81 & 0.29 \\
\hline HAT-P-32b & 11.289 & 0.6 & 58107.213 & 1.02 & 1.95 & 0.05 \\
\hline TrES-1b & 11.76 & 0.7 & 58722.2486 & 3.54 & 1.77 & 0.23 \\
\hline WASP-10b & 12.7 & 1.06 & 58789.2378 & 8.33 & 2.02 & 0.54 \\
\hline WASP-10b & 12.7 & 1.18 & 58721.2001 & 5.15 & 2.86 & 0.48 \\
\hline WASP-10b & 12.7 & 0.95 & 58755.2135 & 4.62 & 2.36 & 0.33 \\
\hline WASP-10b & 12.7 & 1.12 & 58758.3113 & 5.59 & 2.24 & 0.36 \\
\hline WASP-52b & 12.0 & 1.27 & 58771.3086 & 4.82 & 1.95 & 0.4 \\
\hline WASP-52b & 12.0 & 0.97 & 58764.3076 & 4.7 & 2.42 & 0.36 \\
\hline WASP-52b & 12.0 & 0.86 & 58757.3088 & 4.07 & 2.3 & 0.34 \\
\hline WASP-52b & 12.0 & 0.7 & 58038.1527 & 3.41 & 2.85 & 0.37 \\
\hline
\end{tabular}

future follow-up. Assuming root-n scaling, we also estimate the number of 6 inch telescopes, when observing simultaneously, required to detect these transits. We find that a modest-sized network of 166 inch telescopes operated by citizen scientists can increase the number of $3 \sigma$ detections from 5 to 8 . Larger telescopes, or a larger network, would be required to monitor the remaining dimmer and/or shallower targets.

At the conclusion of TESS's primary mission, it is predicted that there will be thousands of transiting exoplanets that are both bright and have large spectral modulation and are therefore ideal for detailed spectroscopic characterization with large platforms (Zellem et al. 2017). This prediction is supported by the population of the current TOIs (Figure 9), which feature planets with large transit depths and bright host stars and, in some cases, large uncertainties in their orbital period and mid-transit time. Some of these targets, due to their bright host stars and large transit depths, are accessible to smaller $(\leqslant 1 \mathrm{~m})$ telescopes. By comparing Figure 9 with Figures 4-8 and Table 5, we can see that many of these targets can be observed with relatively small telescopes at high statistical significance, alleviating the need for larger observatories to keep their transit times fresh, allowing them to followup dimmer targets or smaller transiting exoplanets instead.

\subsection{Advocating for a Network of Small Telescopes Operated by Citizen Scientists}

A network of smaller telescopes $(\gtrsim 16 \geqslant 6$ inch telescopes) could rapidly respond to new discoveries and high priority bright targets with large transit depths and monitor them, allowing larger telescopes to spend their time on other targets (e.g., Earth-sized planets transiting dim M-dwarf stars). Citizen scientists, in particular, provide a unique opportunity to the professional astronomical community: a large number of observers who are eager to aid NASA's mission goals and contribute to the observational needs of the professional community (e.g., Croll et al. 2011; Wiggins \& Crowston 2011; Catlin-Groves 2012; Croll 2012; Franzoni \& Sauermann 2014; Marshall et al. 2015; Kuchner et al. 2016; Burdanov et al. 2018; Collins et al. 2018; Watson \& Floridi 2018).

\subsection{Examples of Successful Citizen Science Efforts}

Specific programs that have leveraged observations by citizen scientists include the OSIRIS-REx missions Target Asteroids! (TA!) and the Astronomical Leagues companion Target NEOs! (TNEO!) measure much needed astrometry and photometry over a wide range of phase angles (Hergenrother \& Hill 2013; Hergenrother et al. 2013, 2014; Lauretta et al. 2017).

A similar telescope network has demonstrated success in supporting observations of asteriod occultation events as well as photometric light curves of asteroids leading to the discovery of moons around asteroids (Timerson et al. 2013; Pravec et al. 2016), the shape and size of large main-belt asteroids (Hanuš et al. 2017), as well as the first ring around an asteroid (BragaRibas et al. 2014).

In addition, the Kilodegree Extremely Little Telescope follow-up network (KELT-FUN) employs citizen scientists, students, and professionals to confirm KELT transit detections (Collins et al. 2018) via increased precision and cadence, higher spatial resolution to search for stellar blends, and spectroscopy to determine the stellar type and properties of transit hosts. KELT-FUN has helped discover $>20$ transiting planets.

Lastly, with the increasing involvement of amateur astronomers in professional-amateur exoplanet collaborations, the American Association of Variable Star Observers (AAVSO) created an Exoplanet Section ${ }^{34}$ in 2015 November. Since then,

\footnotetext{
34 https://www.aavso.org/exoplanet-section
} 


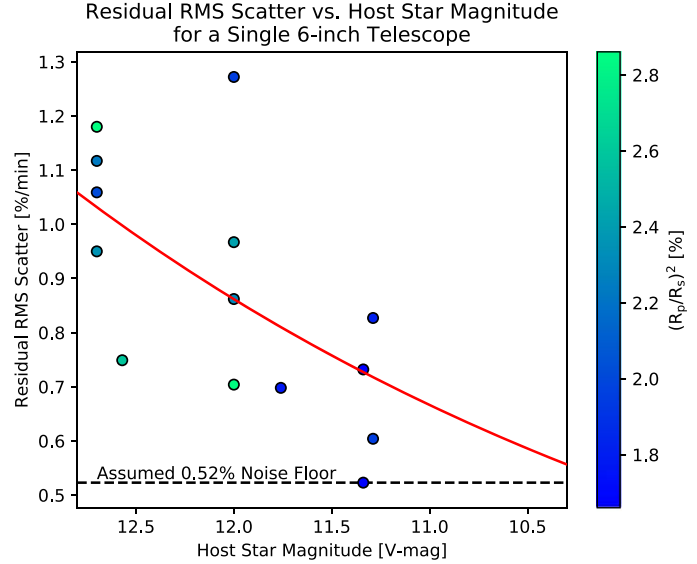

Transit Depth Uncertainty vs. Host Star Magnitude
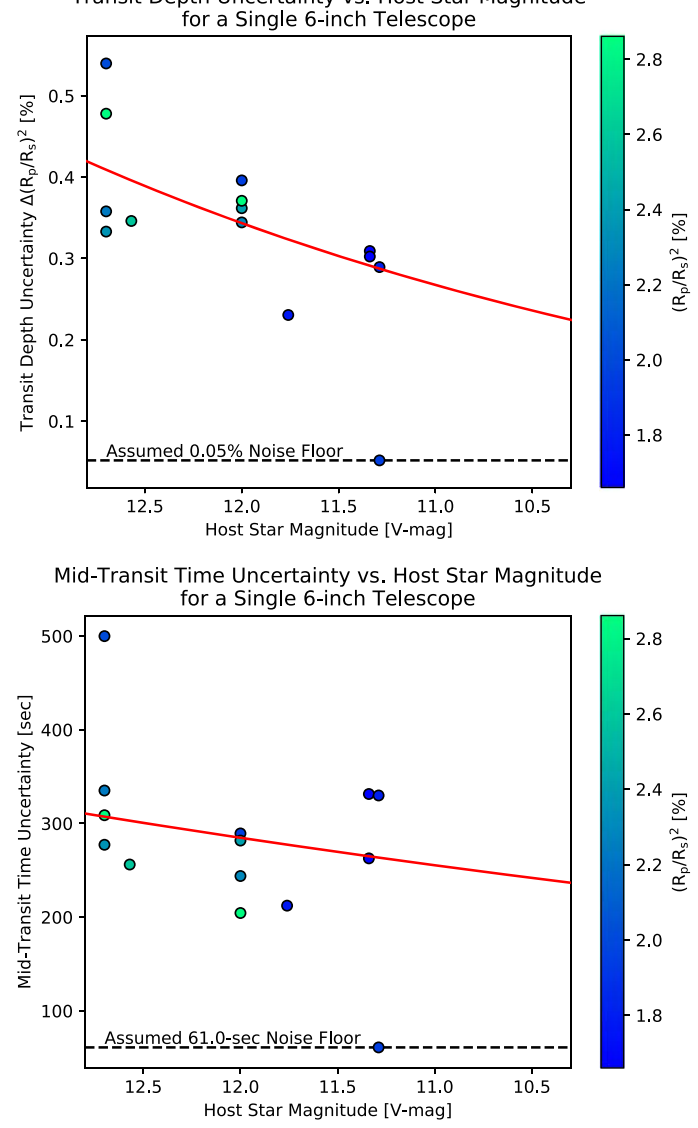

Figure 7. Here we present the measured residual rms scatter (Top), transit depth uncertainty $\Delta\left(R_{p} / R_{s}\right)^{2}$ (Middle), and mid-transit time uncertainty (Bottom) as a function of an exoplanet's host star $V$-magnitude and transit depth $\left(R_{p} / R_{s}\right)^{2}$ for 14 transit observations with a single 6 inch $(15.24 \mathrm{~cm})$ telescope. We fit each data set with a function (red line) that assumes that each precision scales with the square root of the number of photons and conservatively adopt a noise floor that is equal to the minimum measured values. Thus we empirically determine how each parameter varies with an exoplanet's host star $V$-magnitude.

(A color version of this figure is available in the online journal.)
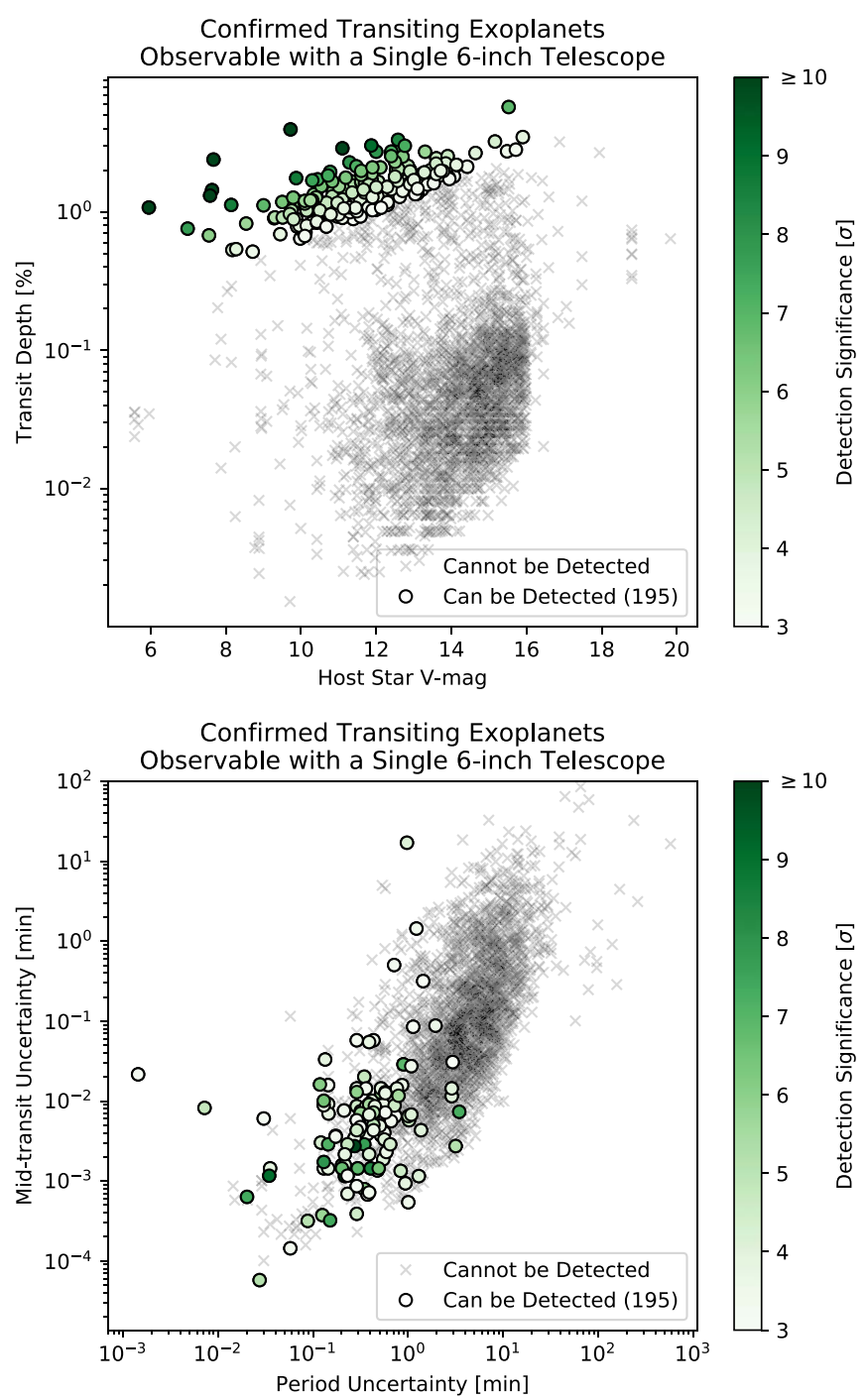

Figure 8. Using the empirically derived functions in Figure 7, we determine that a single 6 inch telescope can observe the transit of 195 currently known exoplanets to $\geqslant 3 \sigma$ (circles), some of with have large timing uncertainties. Here we plot these targets' transit depth $\left(R_{p} / R_{s}\right)^{2}$ vs. their host star $V$-magnitude (Top) and their mid-transit uncertainty vs. their period uncertainty (Bottom) in context with the other confirmed exoplanets that cannot be detected by these small telescopes (gray Xs).

(A color version of this figure is available in the online journal.)

AAVSO members have participated in a major Hubble study whose purpose was to characterize the atmosphere of some 15 confirmed exoplanets, they have been members of the KELTFUN team, and more recently, they have played a key role in the TESS mission. With the latter for example, amateurs are providing follow-up observations of exoplanet candidates in order to help distinguish false positives from true exoplanet transits. An AAVSO Exoplanet Database has also been created 

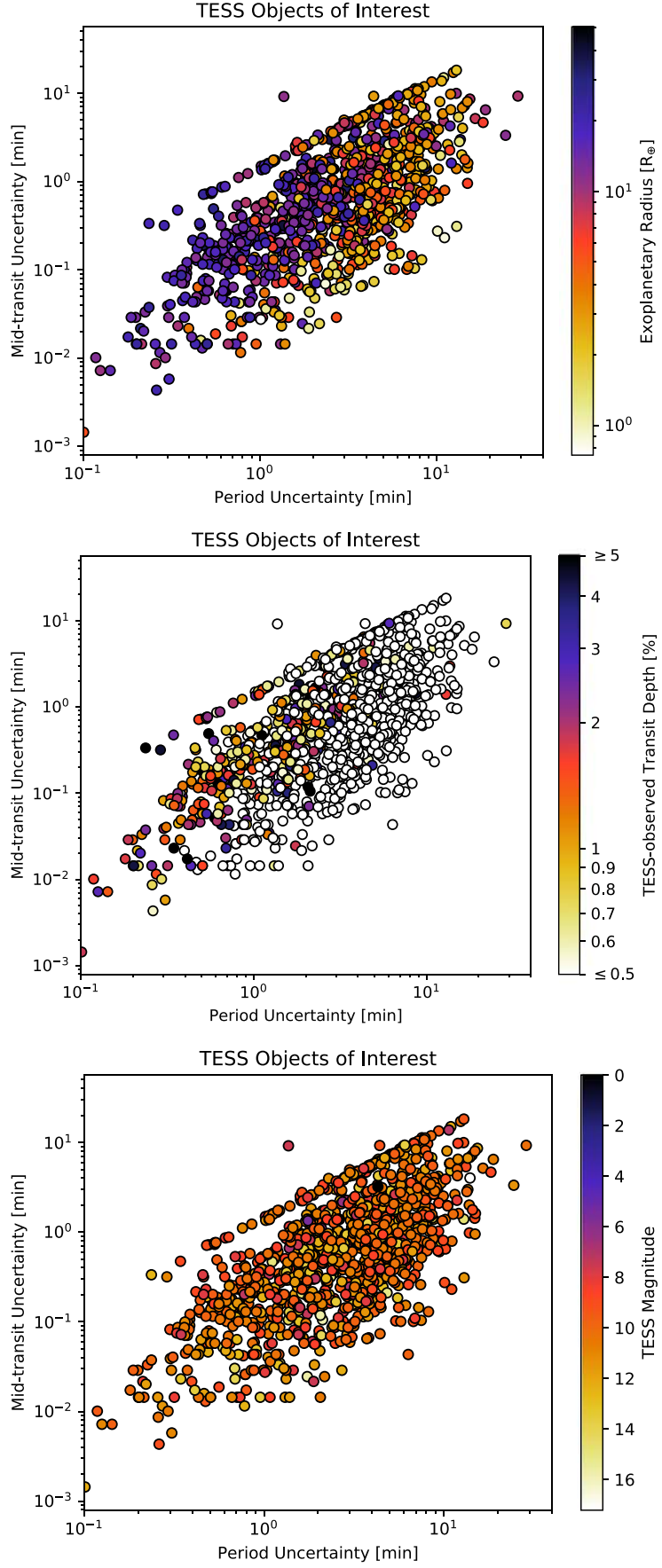

Figure 9. Measured mid-transit uncertainty as a function of orbital period uncertainty and planet radius (top), transit depth (middle), and TESS mag (bottom) of the current TESS Objects of Interest. These targets feature large transits and bright host stars. Some planets, particularly those with Earth-sized radii, have large ( $\sim 10$ minute) uncertainties and could be accessible by smaller telescopes (Figures 4-8 and Table 5). The structure along the top of each plot in the form of a line of points where $\Delta T_{\text {mid }} \approx \Delta P$ (compare to Figure 1 in Kane et al. 2018)

(A color version of this figure is available in the online journal.) as a repository for observations of confirmed exoplanets whose purpose is to help refine their ephemerides, as well as to support TTV studies. Similar efforts are being undertaken by other amateur astronomy groups, such as the British Astronomical Association's Exoplanet Division. ${ }^{35}$

\subsection{Science Returns from a Transiting Exoplanet Citizen Science Network}

\subsubsection{Providing Accurate Transit Ephemerides}

A small-telescope network ( $\gtrsim 16 \geqslant 6$ inch telescopes) operated by citizen scientists dedicated to exoplanet ephemerides maintenance could collect observational data that would be provided to the science community for planning efficient future observations. (This facet in particular is inspired by the Czech Astronomical Society's Transiting Exoplanets and Candidates $^{36}$ program and Exoplanet Transit Database ${ }^{37}$ (Poddaný et al. 2010).) If a particular exoplanet's host star is bright enough, its transit depth is deep enough, and its transit duration is long enough, then a transiting exoplanet is accessible, sometimes at high statistical significance, to smaller telescopes $(\leqslant 1 \mathrm{~m}$; Figure 6$)$.

We estimate the impact of a single 6 inch telescope performing ephemeris maintenance on the Representative TOI Planet via routine, yearly observations. We first forwardpropagate the Representative TOI mid-transit uncertainty using Equation (3) and estimate the uncertainty on the mid-transit time with a single 6 inch telescope (Figure 7). Using these two measured mid-transit times and uncertainties (one from TESS, the other from a single 6 inch telescope) and the orbital period and uncertainty measured by TESS, we solve Equation (1) for the planet's period $P$ and mid-transit time $T_{0}$ and associated uncertainties using a MCMC (e.g., Ford 2005). We then simulate an observing campaign of yearly revisits by the single 6 inch telescope, taking into account its cumulative (previous) observations. We present the results of these simulations in Figure 10 and find that even a single 6 inch telescope can powerfully keep the Representative TOI Planet fresh for $\sim 1 \mathrm{yr}$, ensuring efficient follow-up by JWST, ARIEL, and an Astro2020 mission.

We next perform a detailed analysis of the 1000-planet population used by the CASE team for their independent study of ARIEL's capabilities (Zellem et al. 2019b). This target list is approximately the same size as the ARIEL mission Tier-1 sample (Tinetti et al. 2016, 2018; Edwards et al. 2019), contains the currently known transiting exoplanets and those predicted to be discovered with TESS (Sullivan et al. 2015),

\footnotetext{
35 https://britastro.org/section_front/15474

36 http://var2.astro.cz/EN/tresca/index.php

${ }^{37}$ http://var2.astro.cz/ETD/
} 


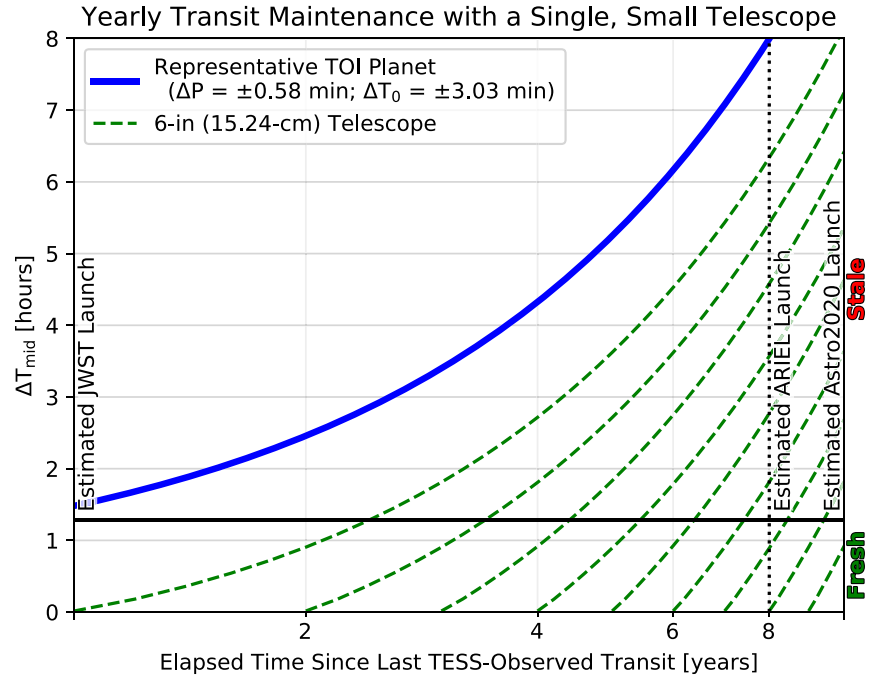

Figure 10. The mid-transit uncertainty as a function of the elapsed time since the last TESS observation of the Representative TOI Planet (thick blue solid line) and cumulative observations with a single 6 inch telescope, repeated yearly (dashed green lines). Yearly, routine transit maintenance by a single 6 inch telescope can keep the Representative TOI Planet fresh for about a year at a time.

(A color version of this figure is available in the online journal.)

and is chosen by a figure of merit that prioritizes planets with large scale heights orbiting bright stars (Equation (5); Cowan et al. 2015; Zellem et al. 2017; Goyal et al. 2018; Kempton et al. 2018; Morgan et al. 2019).

To estimate the time saved by a citizen science ground-based monitoring effort, we adopt the mid-transit times, orbital periods, and their associated uncertainties from the NASA Exoplanet Archive (Akeson et al. 2013) for the known planets. For the predicted TESS targets, we estimate each planet's midtransit and period uncertainties from the current confirmed TESS-discovered exoplanets. We find that the mid-transit and period uncertainties of these planets do not scale with their host stars' $V$-magnitudes (Figure 11), potentially suggesting that these objects are in a systematic-dominated regime. Assuming the photon-limited regime, one would reasonably expect the measured precisions to scale with the number photons and thus the number of repeat observations. However, since the number of observations (by TESS and by follow-up observations from the TFOP) that went into determining these values are not immediately available to us, we have conservatively adopted the median uncertainties of $64.66 \mathrm{~s}$ for $\Delta T_{0}$ and $26.35 \mathrm{~s}$ for $\Delta P$, regardless of the host star's $V$-magnitude.

For each predicted TESS target, we adopt an ephemeris of the end of TESS's nominal mission lifetime ( 2020). Then, using Equation (3), we propagate the uncertainty of each planet's mid-transit time at six different epochs: the beginning and end of the nominal missions for JWST, ARIEL, and an Astro2020 Decadal mission, such as HabEx (Gaudi et al. 2018), LUVOIR (The LUVOIR Team 2018), or the Origins
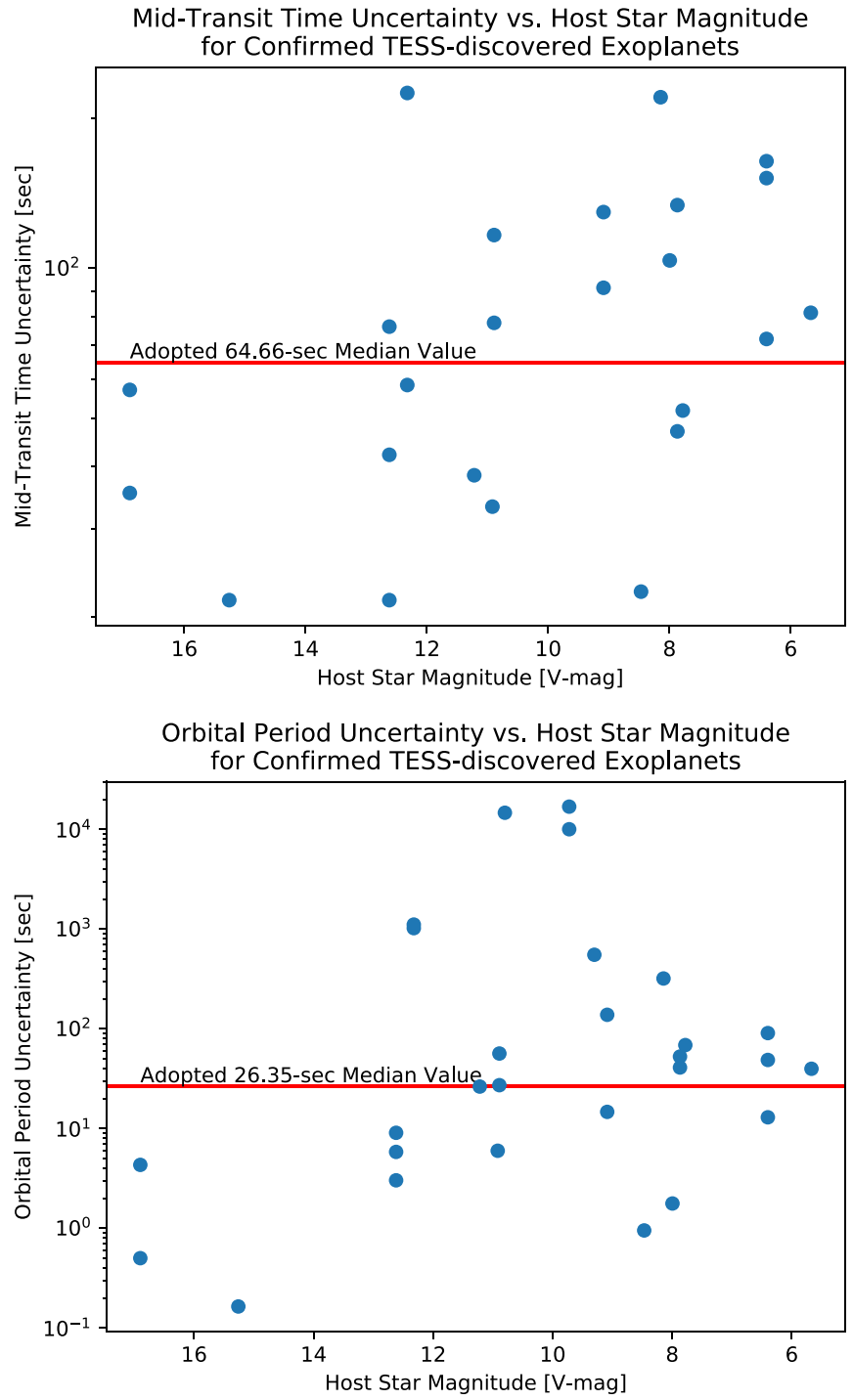

Figure 11. Similar to Figure 7, except for the confirmed TESS-discovered exoplanets. These planets seem to be in a systematic-dominated regime, as evidenced by their non-correlation between the mid-transit and orbital period uncertainties and the host star $V$-magnitude. Therefore, we conservatively adopt the median uncertainties of $64.66 \mathrm{~s}$ for $\Delta T_{0}$ and $26.35 \mathrm{~s}$ for $\Delta P$, regardless of the host star's $V$-mag.

(A color version of this figure is available in the online journal.)

Space Telescope (Battersby et al. 2018). For each planet, we conservatively adopt a covariance term $\Delta P \Delta T_{0}=0$ to provide a lower-limit estimate. We then simulate a mid-transit measurement of a single 6 inch telescope at each of these six epochs by estimating their mid-transit uncertainties from their host star $V$-magnitude, as described in detail in Section 4 and Figure 7 and use a MCMC to solve for a new mid-transit ephemeris (similar to the procedure described in detail in Section 4.3.1), taking into account the 6 inch telescope observation as well as either the ephemeris reported on the NASA Exoplanet Archive for the real planets or the median 


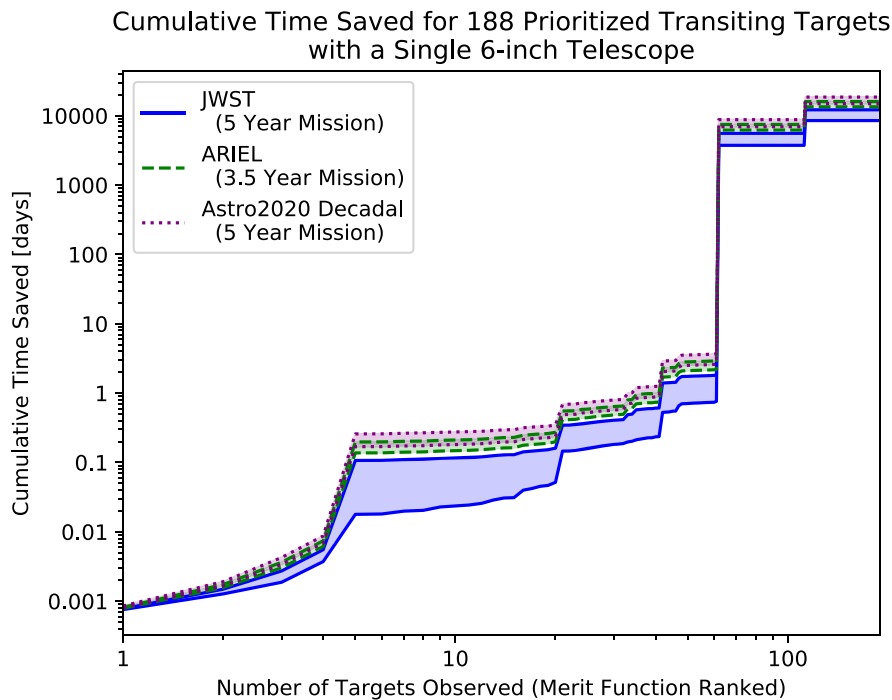

Cumulative Time Saved for 507 Prioritized Transiting Targets with 16 6-inch Telescopes

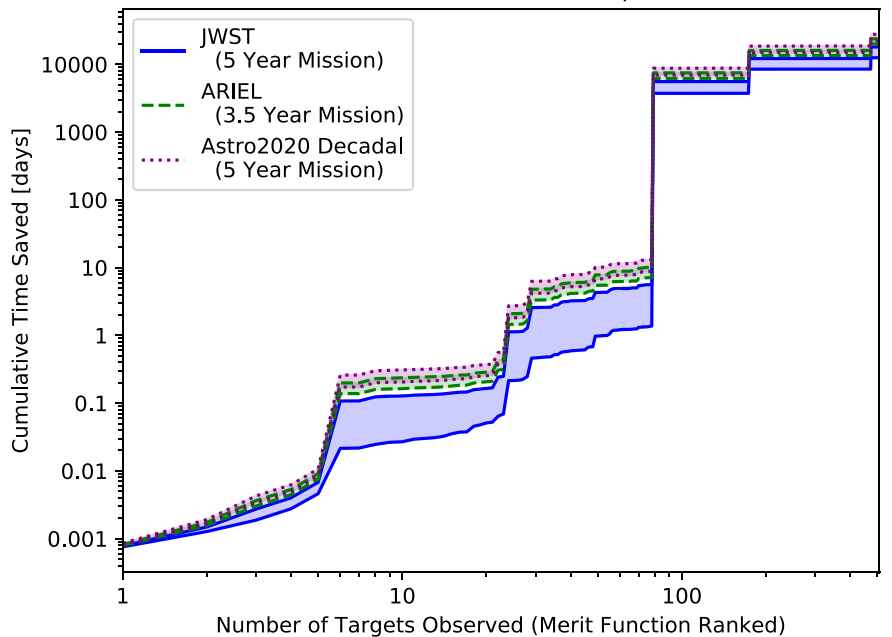

Cumulative Time Saved for 186 Prioritized Transiting Targets with a Single 6-inch Telescope

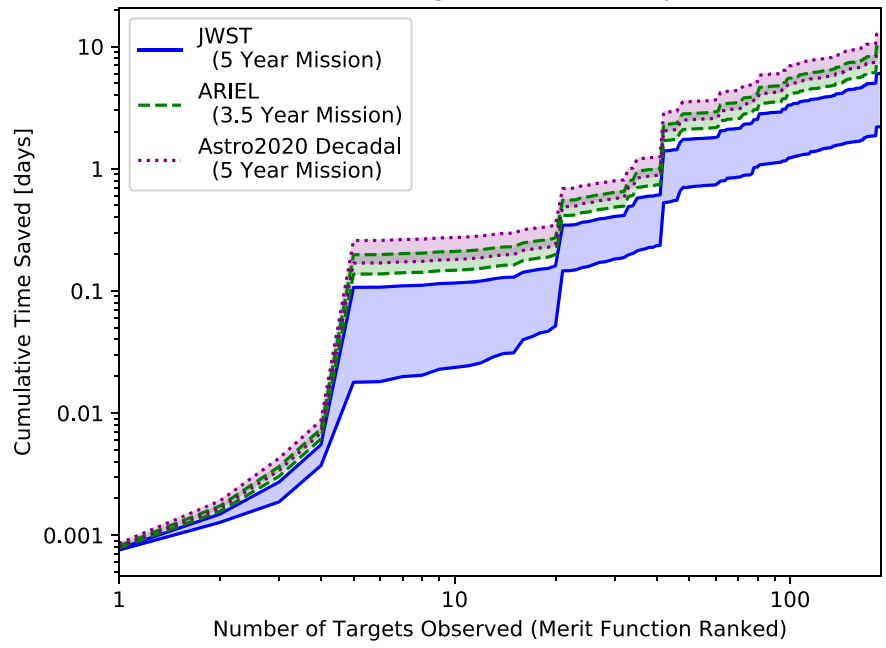

Cumulative Time Saved for 504 Prioritized Transiting Targets with 16 6-inch Telescopes

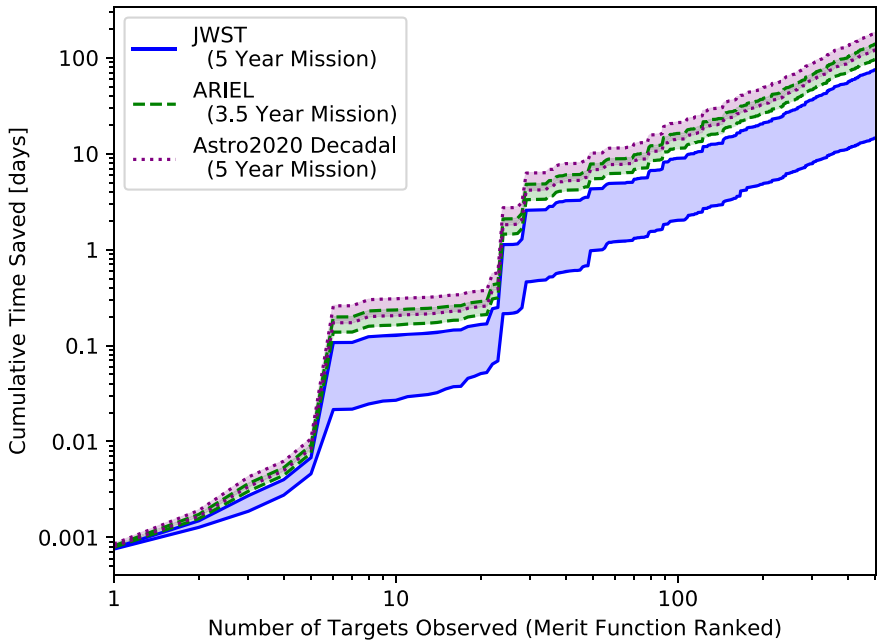

Figure 12. The cumulative time saved with single-visit transit maintenance on a single 6 inch telescope (top row) and a network of 166 inch telescopes (bottom row) for JWST (solid blue lines), ARIEL (dashed green lines), and an Astro2020 Decadal Mission (dotted purple lines). The targets are drawn from the 1000 targets bestsuited for transit spectroscopy (targets are relatively ranked by their scale height and host star magnitude and are a mix of the currently known exoplanets and those predicted to be discovered with TESS; Zellem et al. 2017) and are then filtered for $3 \sigma$ detections of the transit. The shaded regions show the time saved over the entire lifetime of each mission. Transit maintenance with even a single 6 inch telescope can save on the order of $\sim 10,000$ days for both JWST and ARIEL while a network of 166 inch telescopes could save on the order $\sim 5000$ days and $\sim 20,000$ days, respectively. The comparatively large "jumps" in the data (left column) are where the published transit times have large uncertainties, therefore a single 6 inch telescopic observation can significantly refine the times for these targets. When the two (top row) and three (bottom row) targets with the largest uncertainties are removed from this target list (right column), we more conservatively find that the time saved by a single 6 inch telescope is on the order of $\sim 5$ days, while the time saved by a network of 166 inch telescopes is on the order of $\sim 80$ days.

(A color version of this figure is available in the online journal.)

TESS uncertainties (Figure 11) for the predicted TESS targets. The time saved by a small telescope is then the difference between the transit time uncertainty $\Delta T_{\text {mid }}$ propagated from values listed on the NASA Exoplanet Archive or simulated from TESS and the new reduced uncertainty due to an observation by a single 6 inch telescope. We estimate that even a single 6 inch telescope performing transit maintenance could observe the transit of 188 exoplanets to $3 \sigma$ and thus save $\sim 10,000$ days for both JWST and ARIEL (Figure 12, Top Left) while a network of 166 inch telescopes could observe 507 exoplanets and therefore save $\sim 5000$ days or JWST during a 200 planet survey and $\sim 20,000$ days for ARIEL's 1000planet survey (Figure 12, Bottom Left). The "jumps" in the left plots in Figure 12 represent planets that have large ephermeris 
unceratinties listed on the NASA Exoplanet Archive. Therefore, a single observation by one 6 inch telescope can greatly decrease these uncertainties, resulting in a large savings of time. If we remove the two (top row) and three (bottom row) exoplanets with the largest ephemeris uncertainties from the target list, we still find that a single 6 inch telescope can save future missions $\sim 5$ days while a network of 166 inch telescopes can save future missions $\sim 80$ days (Figure 12 right column).

\subsubsection{Measuring Planetary Masses and Discovering Additional Companions}

If citizen scientists continue to monitor targets over a large baseline, they could enable the measurement of TTVs, the discovery of new exoplanets, and aid in the calculation of the masses of known planets (e.g., Agol et al. 2005; Holman \& Murray 2005; Dalba \& Muirhead 2016). Past detections of TTVs have primarily come from the Kepler mission due to its long-term photometric monitoring of the same field of stars for $4 \mathrm{yr}$ (Borucki et al. 2010). One large constraint on the search for timing variations is TESS' significantly shorter baseline compared to Kepler. Only select areas of the sky will have data for more than 27.4 days and, based on prior studies, $\sim 20$ transits are generally needed to ensure a unique orbit solution when interpretting TTVs (Nesvorný \& Morbidelli 2008). This requirement limits the TTV search to planets in compact orbits if they have only one sector of TESS data. For multi-planet systems, the average period of an inner planet is $\sim 10$ days (Mulders et al. 2018) suggesting follow-up is necessary even to achieve three transit measurements to confirm the planet let alone detect a TTV.

One of the primary objectives for the TESS Followup Program (TFOP) is to help achieve TESS's Level 1 requirement to measure masses for 50 planets smaller than $4 R_{\oplus} \cdot{ }^{38} \mathrm{We}$ estimate that this radius constraint corresponds to a mass of $15_{-5}^{+7} M_{\oplus}$ based on a mass-radius relation derived from a Gaussian Process (using the gpy Python package; GPy 2012) of planets with measured masses $\left(M_{s}<2 M_{\odot}\right)$, radii, and orbital periods $(1 \leqslant P \leqslant 100$ days) from the NASA Exoplanet Archive (Akeson et al. 2013). The Gaussian Process uses 524 exoplanets to determine a mass-radius relationship and uncertainty (Pearson 2019). We estimate that a $15 M_{\oplus}$ planet with a short orbital period corresponds to a radial velocity semi-amplitude signal on the order of $\sim$ few $\mathrm{m} \mathrm{s}^{-1}$, which currently pushes the limits of modern instruments (e.g., Fischer et al. 2016; Delisle et al. 2018).

However, a network of smaller telescopes can provide additional transit measurements to contribute to the TFOP and community efforts (e.g., KOINetwork; von Essen et al. 2018; Freudenthal et al. 2019) to determine planetary masses via TTV

\footnotetext{
38 https://tess.mit.edu/followup/
}

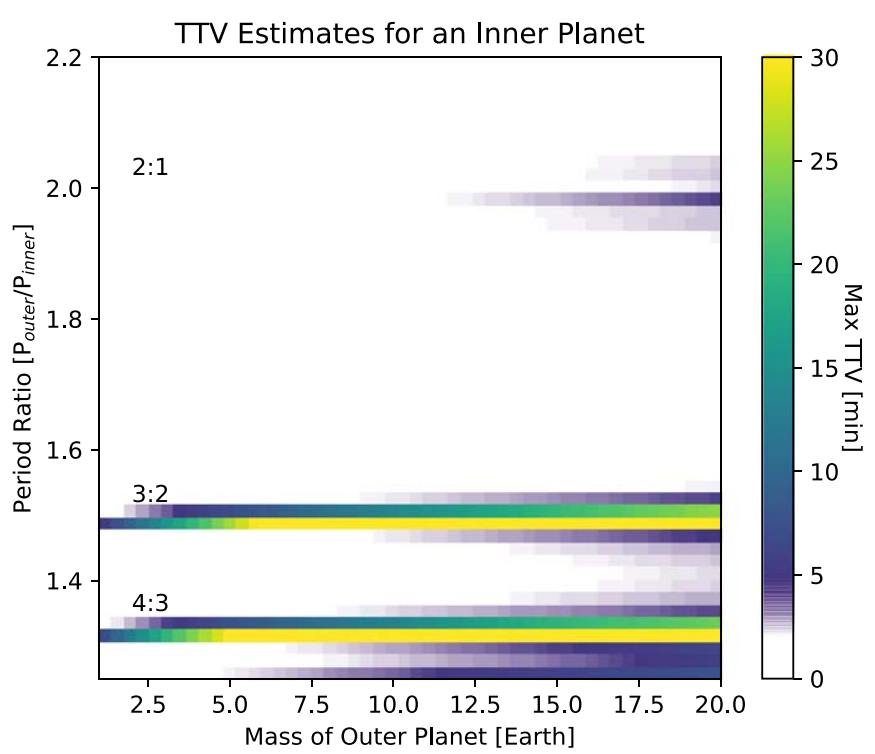

Figure 13. Transit timing variations of an inner most planet estimated with various companion planets. The $N$-body simulations (https://github.com/ pearsonkyle/Nbody-AI) use a stellar mass of $1.26 M_{\odot}$ and the innermost planet is the Representative TOI Planet with an orbital period of 3.85288 days with a mass of $0.23 M_{\text {Jupiter }}$. The fixed parameters are median values computed from the TESS TOI catalog. The second, outer companion planet in the system has varying masses and orbital periods. Each grid point is an $\mathrm{N}$-body simulation run for 180 days, with the colors being mapped to the maximum perturbation after performing a linear fit to the transit times of the inner planet.

(A color version of this figure is available in the online journal.)

measurements (Figures 13 and 14), thus helping to achieve one of TESS's primary objectives. Therefore, even if all of the transiting exoplanets observed by TESS have ephemeris precisions high enough where they are not at risk of becoming stale, a network of small telescopes are still necessary to establish long baselines to search for TTVs or additional transiting planets.

The fraction of false positives planet candidates can be reduced by requiring at least three self-consistent transit detections instead of a single one (Petigura et al. 2013; Burke et al. 2014; Rowe et al. 2015; Coughlin et al. 2016). Even if the mutual inclination of multi-planet systems yield non-transiting companions their orbit and mass can still be constrained through TTV measurements of at least one transiting planet (e.g., Pearson 2019).

The TTV of existing multi-planet systems is estimated and reported in Table 6. This subset of planets is ranked by the maximum TTV amplitude calculated from 20 simulated transit epochs. Only planets in the NASA Exoplanet Archive (Akeson et al. 2013) with periods less than 60 days and host stars $<2 M_{\odot}$ are used. These targets could benefit from TESS high cadence observations or follow-up observations in order to constrain the parameters of the perturbing planet. For example, a single 6 inch telescope could achieve a mid-transit precision $\Delta T_{0}$ of 1.02 minutes for even a $11.289 \mathrm{~V}$-mag star (Figure 5), 


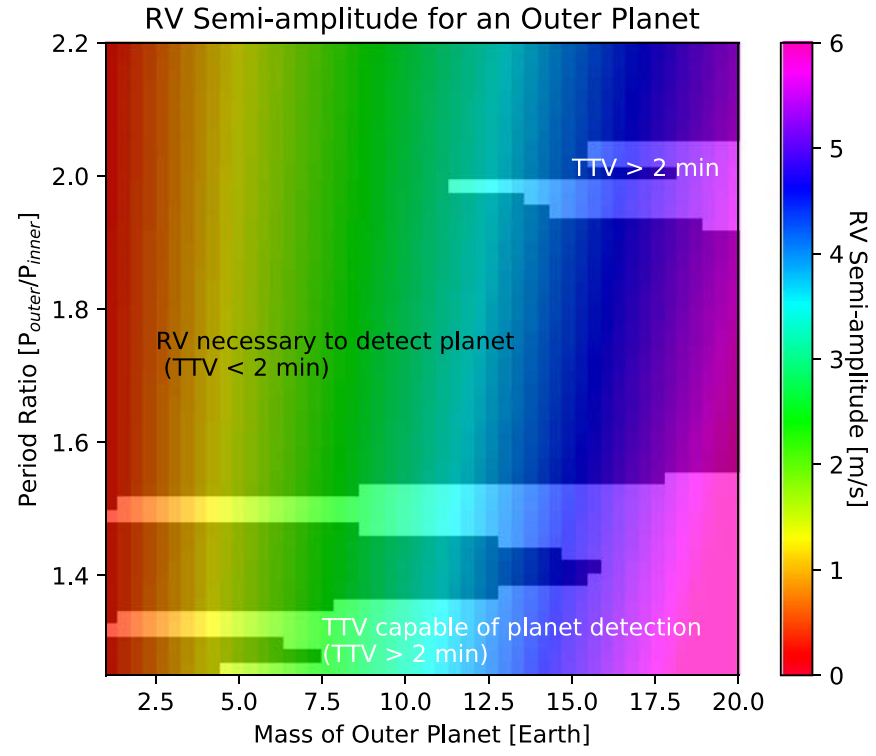

Figure 14. Estimated radial velocity semi-amplitude signals for an outer planet with 1-20 $M_{\oplus}$ and an orbital period 1.25-2.2× the length of its inner planet, here assumed to be the Representative TOI Planet (3.85288 days). Currently, Earth-sized planets are difficult to detect with radial velocity instruments since the signals are only a $\sim$ few $\mathrm{m} \mathrm{s}^{-1}$. However, it is possible to detect Earth-sized planets using TTV measurements but only at certain orbit configurations, particularly those near close orbital resonances. The darker region is based on Figure 13 and masks a portion of the parameter space where TTVs are $>2$ minutes and thus could be accessible with smaller ground-based telescopes. Unmasked regions are where the TTV signal is $<2$ minutes, thereby requiring radial velocity measurements.

(A color version of this figure is available in the online journal.)

allowing the opportunity for even relatively small telescopes to measure TTVs (particularly those listed in Table 6) to high significance. Multi-planet systems are common with somewhere between $\sim 50 \%$ and $70 \%$ of exoplanets being in a multiplanet system. The other fraction could be single planet systems or multi-planet systems with higher mutual inclinations. Not all multi-planet architectures yield observable TTVs though (Figure 13). Based on Kepler data, the spacing between adjacent planets follows a wide distribution with a peak at an orbital period ratio of 1.8 (Mulders et al. 2019). Observational biases can reduce this ratio since it is easier to measure a TTV for a more compact orbit than for those far apart.

\subsubsection{Helping Confirm Long-period Planets}

This network of observers could also support the TFOP effort to continue to monitor TESS fields, particularly those which will be observed by TESS for only 27.4 days, helping to confirm planets with long orbital periods $(P>27.4$ days $)$ which would have only one TESS-observed transit. A citizen scientist could be directed to a particular field which contains both a known transiting planet in need of maintenance and a potential long-period planet, since small telescopes are capable of having large fields-of-view (e.g., a 6 inch MicroObservatory image is 0.94 by $0.72,3.4 \times$ the angular area of the full Moon). Thus an observer could both perform ephemeris maintenance on the known transit planet while simultaneously monitoring the other host star for potential transits.

\subsubsection{Spatially Resolving Stellar Blends}

TESS' pixel scale is $21^{\prime \prime}$, raising the possibility that an object that appears to be a single star in a TESS image is rather a blend of multiple stars (Figure 15). Therefore due to the dilution by additional stars within the pixel, an observed transit would appear shallower than it truly is, thereby altering the physical interpretation of the planet (e.g., Crossfield et al. 2012; Bergfors et al. 2013; Stevenson et al. 2014; Ciardi et al. 2015; Collins et al. 2018; Colón et al. 2018). While spatial resolution has typically been conducted on larger platforms, smaller telescopes could probe fields at $\gtrsim 5 \times$ higher spatial resolution than TESS, assuming seeing-limited observations on the order of $1^{\prime \prime}-4^{\prime \prime}$.

But even achieving spatial resolutions that are plate-scale limited (rather than seeing-limited) are still useful for identifying stellar blends. For example in the top panel of Figure 15, we present a subset of a single image by a 6 inch MicroObservatory telescope of the stars TYC 3280-846-1 and TYC 3280-697-1, which have $V$-magnitudes of 9.83 and 10.68, respectively (Wenger et al. 2000). Despite the image not being seeing-limited (the plate scale is $5.21 \mathrm{arcsec} / \mathrm{pixel}$ ) and imperfect tracking over the course of the exposure (causing the oblong shape of the stars), the MicroObservatory is able to distinctly resolve these two stars. However, when we bin this image to the spatial resolution of TESS ( $21 \mathrm{arcsec} / \mathrm{pixel})$, the two stars, which are separated by $21{ }^{\prime \prime} 5$, blend together and appear as a single source. Assuming that TYC 3280-846-1 is similar in size to the Sun and hosts a Jupiter-sized transiting exoplanet, the MicroObservatory would observe a $1 \%$ transit depth. However since TESS would blend the two stars, TESS would observe a transit depth of $0.3 \%$ (calculated using Equation (4) in Ciardi et al. 2015), and would potentially incorrectly classify this hot Jupiter as a sub-Neptune.

\subsubsection{Characterizing Epoch-to-epoch Stellar Variability}

If sufficiently large, stellar activity (spots and/or plages) can change the observed transit signal, thereby altering the retrieved physical properties of the planet itself (Alonso et al. 2008; Pont et al. 2008; Silva-Valio 2008; Czesla et al. 2009; Wolter et al. 2009; Agol et al. 2010; Berta et al. 2011; Carter et al. 2011; Désert et al. 2011; Sing et al. 2011; Narita et al. 2013; Fraine et al. 2014; McCullough et al. 2014; Oshagh et al. 2014; Barstow et al. 2015; Damasso et al. 2015; Zellem et al. 2015, 2017; Rackham et al. 2017, 2018; Zhang et al. 2018). Epoch-to-epoch host star variability has been monitored and quantified by long-term ground-based photometric observations. For example, data from the Tennessee State University 
Table 6

TTV Estimates for Exoplanet Systems that are Ideal for TTV Measurements with Small Ground-based Observatories

\begin{tabular}{|c|c|c|c|c|c|c|c|c|}
\hline Planet & $\begin{array}{c}\text { Planet Mass } \\
\qquad\left(M_{\oplus}\right)\end{array}$ & $\begin{array}{l}\text { Period } \\
\text { (days) }\end{array}$ & Eccentricity & $\begin{array}{c}\text { Stellar Mass } \\
\qquad\left(M_{\odot}\right)\end{array}$ & $V$-mag & $\begin{array}{c}\text { TTV } \\
\text { (minutes) }\end{array}$ & $\begin{array}{l}\text { Discovery } \\
\text { Paper }\end{array}$ & $\begin{array}{l}\text { Parameter } \\
\text { References }\end{array}$ \\
\hline Kepler-30 b & 11.44 & 29.334 & 0.046 & 0.99 & 15.403 & 746.9 & Fabrycky et al. (2012) & Sanchis-Ojeda et al. (2012) \\
\hline KOI-142 b & 8.58 & 10.954 & 0.026 & 0.96 & 13.11 & 189.5 & Nesvorný et al. (2013) & Nesvorný et al. (2013) \\
\hline Kepler-9 c & 54.35 & 38.91 & 0.005 & 1.07 & 13.803 & 127.8 & Holman et al. (2010) & Holman et al. (2010) \\
\hline $\mathrm{K} 2-266 \mathrm{~d}$ & 9.4 & 14.697 & 0.007 & 0.69 & 11.808 & 122.8 & Rodriguez et al. (2018) & Rodriguez et al. (2018) \\
\hline Kepler-223 c & 5.1 & 9.846 & 0.003 & 1.12 & 15.344 & 77.0 & Rowe et al. (2014) & Mills et al. (2016) \\
\hline TRAPPIST-1 f & 0.68 & 9.207 & 0.004 & 0.08 & 18.8 & 32.3 & Gillon et al. (2016) & Gillon et al. (2017) \\
\hline Kepler-36 b & 4.45 & 13.84 & 0.002 & 1.07 & 11.866 & 30.8 & Carter et al. (2012) & Carter et al. (2012) \\
\hline Kepler-11 f & 1.91 & 46.689 & 0.001 & 0.96 & 14.2 & 26.1 & Lissauer et al. (2011) & Lissauer et al. (2013) \\
\hline $\mathrm{K} 2-285 \mathrm{~d}$ & 6.5 & 10.456 & 0.003 & 0.83 & 12.03 & 21.0 & Palle et al. (2018) & Palle et al. (2018) \\
\hline Kepler-30 c & 638.81 & 60.323 & 0.0 & 0.99 & 15.403 & 20.2 & Fabrycky et al. (2012) & Sanchis-Ojeda et al. (2012) \\
\hline $\mathrm{K} 2-32 \mathrm{~d}$ & 10.3 & 31.715 & 0.002 & 0.86 & 12.31 & 19.7 & Dai et al. (2016) & Petigura et al. (2017) \\
\hline Kepler-79 e & 4.13 & 81.066 & 0.0 & 1.17 & 13.914 & 12.9 & Rowe et al. (2014) & Jontof-Hutter et al. (2014) \\
\hline $\mathrm{K} 2-24 \mathrm{c}$ & 15.4 & 42.339 & 0.001 & 1.07 & 11.07 & 11.6 & Petigura et al. (2016) & Petigura et al. (2018) \\
\hline $\mathrm{K} 2-24 \mathrm{~b}$ & 19.0 & 20.89 & 0.003 & 1.07 & 11.07 & 11.5 & Petigura et al. (2016) & Petigura et al. (2018) \\
\hline $\mathrm{K} 2-32 \mathrm{c}$ & 12.1 & 20.66 & 0.001 & 0.86 & 12.31 & 11.4 & Dai et al. (2016) & Petigura et al. (2017) \\
\hline KOI-94 c & 15.57 & 10.424 & 0.003 & 1.28 & 12.205 & 10.9 & Weiss et al. (2013) & Weiss et al. (2013) \\
\hline Kepler-11 e & 7.95 & 32.0 & 0.001 & 0.96 & 14.2 & 10.8 & Lissauer et al. (2011) & Lissauer et al. (2013) \\
\hline Kepler-11 d & 7.31 & 22.684 & 0.001 & 0.96 & 14.2 & 9.7 & Lissauer et al. (2011) & Lissauer et al. (2013) \\
\hline Kepler-79 c & 6.04 & 27.403 & 0.001 & 1.17 & 13.914 & 7.7 & Xie (2013) & Jontof-Hutter et al. (2014) \\
\hline Kepler-79 d & 6.04 & 52.09 & 0.0 & 1.17 & 13.914 & 7.6 & Rowe et al. (2014) & Jontof-Hutter et al. (2014) \\
\hline $\mathrm{K} 2-285 \mathrm{e}$ & 10.7 & 14.763 & 0.001 & 0.83 & 12.03 & 6.5 & Palle et al. (2018) & Palle et al. (2018) \\
\hline TRAPPIST-1 d & 0.41 & 4.05 & 0.005 & 0.08 & 18.8 & 6.5 & Gillon et al. (2016) & Gillon et al. (2017) \\
\hline Kepler-18 c & 17.16 & 7.642 & 0.002 & 0.97 & 13.549 & 6.1 & Cochran et al. (2011) & Cochran et al. (2011) \\
\hline $\mathrm{K} 2-266 \mathrm{c}$ & 0.29 & 7.814 & 0.001 & 0.69 & 11.808 & 2.3 & Rodriguez et al. (2018) & Rodriguez et al. (2018) \\
\hline TRAPPIST-1 e & 0.62 & 6.1 & 0.004 & 0.08 & 18.8 & 2.1 & Gillon et al. (2016) & Gillon et al. (2017) \\
\hline Kepler-101 c & 3.18 & 6.03 & 0.001 & 1.17 & 13.8 & 2.0 & Rowe et al. (2014) & Bonomo et al. (2014) \\
\hline
\end{tabular}

Celestron-14 $(0.36 \mathrm{~m})$ AIT at Fairborn Observatory in southern Arizona (e.g., Henry 1999; Eaton et al. 2003) has been used to quantify the time-varying activity of the hot Jupiter host star HD 189733b. These data have then been used to confirm that the host star's activity does not impact the planet's phase curve (Knutson et al. 2012) nor its multi-epoch eclipse measurements (Kilpatrick et al. 2019) as observed with Spitzer/IRAC. While it is estimated that epoch-to-epoch stellar variations will not influence the observed planetary signal in most cases, if the observations are at sufficiently high precision (as is typically the case for bright targets), the transit depth is large, and the star is active enough, stellar variability could impact the observed transit signal (see Figure 3 in Zellem et al. 2017). Epoch-to-epoch stellar activity $\gtrsim 0.2 \%$ could begin to effect high precision observations (e.g., with JWST) of transit depths on the order of $\sim 1 \%$ (see Figure 3 in Zellem et al. 2017).

Fortunately, small telescopes have the capability of high precision observations even for relatively dim stars. From Figure 7 Top we estimate that for a $11.3 \mathrm{~V}$-mag star, a 6 inch telescope could achieve a per-minute precision of $0.67 \% \pm$ $0.12 \%$. Note that this value and its associated uncertainty is calculated from four total observations by the same 6 inch 

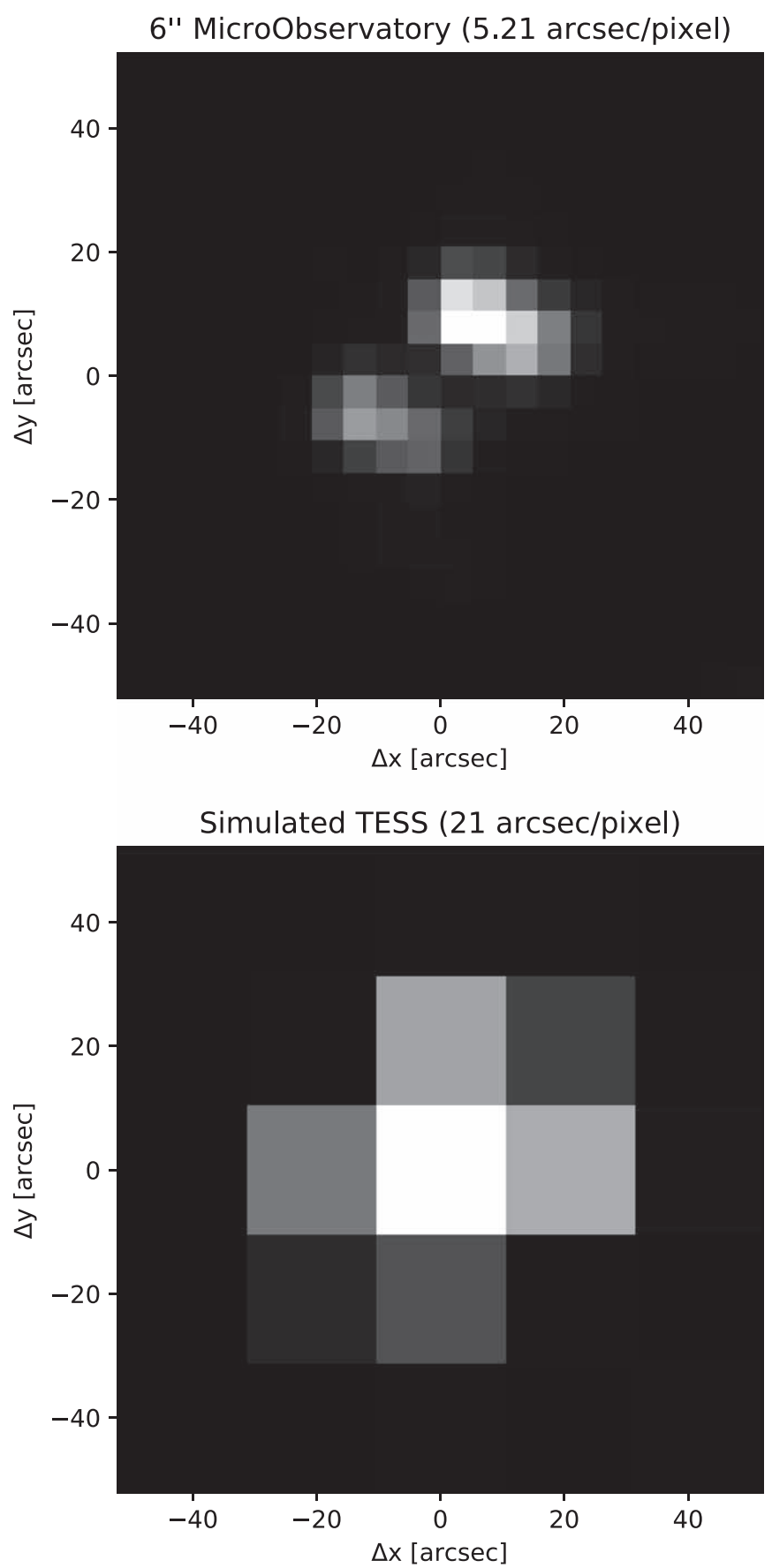

Figure 15. This subset of a 6 inch MicroObservatory image (Top) clearly distinguishes between two stars, which are separated by 21 ". 5 . However, due to TESS' relatively large plate scale (21 arcsec/pixel), these two stars blend together and appear as one source (Bottom). If a planet were to be discovered orbiting either of these stars, TESS would observe a diluted transit, thereby misidentifying the planet's radius and therefore its type.

telescope: two independent observations of HAT-P-20b and two independent observations of HAT-P-32b taken over four different nights (Table 5) and thus takes into account night-tonight variations. A 6 inch telescope could therefore measure
$2.1 \%$ variability to $3 \sigma$ on a $11.3 \mathrm{~V}$-mag star in only a minute of observing. Using the observations of WASP-52b taken over four separate nights by the same 6 inch telescope, we estimate that a single 6 inch telescope can achieve a per-minute precision of $0.95 \% \pm 0.21 \%$ for a $12 \mathrm{~V}$-mag star; similarly using the observations of WASP-10b taken over four separate nights by the same 6 inch telescope, we estimate a per-minute precision of $1.08 \% \pm 0.08 \%$ for a $12.7 \mathrm{~V}$-mag star. Thus, an amateur astronomer could monitor exoplanet host stars for time-varying flux suggesting stellar variability before or after their transit observations and could improve their measured precision by binning over time.

\section{Results and Discussion}

A network of small telescopes (here defined as $\gtrsim 16 \geqslant 6$ inch telescopes; see Section 4 and Table 5) can crucially aid future transiting exoplanet observations (with, e.g., HST JWST, ARIEL, Astro2020 Decadal, and larger ground-based platforms; Figures 10-12) by providing up-todate and accurate transit ephemerides of planets discovered with ground- and space-based surveys. Transit maintenance with even a single 6 inch telescope can save up to $\sim 10,000$ days for both JWST and ARIEL whereas a network of $166 \mathrm{inch}$ telescopes could save on the order $\sim 5000$ days for a 200-planet JWST survey and $\sim 20,000$ days for a 1000-planet ARIEL survey (Figure 12). Thus while transit ephemeris uncertainties do not prevent the accomplishment of any of these missions, precise mid-transit time predictions would enable each mission to act more efficiently and potentially achieve even more science.

This network, in addition to helping confirm long-period $(P>27$ days) planets and spatially resolving stellar blends, could use TTVs in multi-planet systems to measure planetary masses, thereby contributing to the achievement of one of TESS' Level 1 Requirements, and discover new planets. Our $\mathrm{N}$-body simulations estimate that TTVs could be as large as 30 minutes or more ( 0.02 days), which can be achieved with high significance with even a single 6 inch ground-based telescope (Figures 4 and 5 and Table 5; see also Fowler 2019).

Lastly, this network of small telescopes could monitor exoplanet host stars for variability due to, e.g., spots and plages. Epoch-to-epoch variability on the order of even $0.2 \%$ can effect JWST observations of exoplanets with a $\sim 1 \%$ transit depth (Zellem et al. 2017). We estimate that a single 6 inch telescope could measure the variability of a $11.3 \mathrm{~V}$-mag star with a per-minute precision of $0.67 \% \pm 0.12 \%$.

\section{Ramifications for Other Platforms and Surveys}

While transit maintenance could be conducted with TESS via an extended mission (Bouma et al. 2017; Huang et al. 2018; Dalba et al. 2019) or professional observatories (e.g., Benneke et al. 2017), a large ground-based collaboration of smaller 
telescopes $(\leqslant 1 \mathrm{~m})$ could follow-up hundreds of bright targets with large transit depths. Such a network would free-up precious time on TESS, other missions, and larger groundbased telescopes that could be instead dedicated to observing new fields (e.g., the ecliptic), comparatively under-sampled fields (e.g., the 27.4 days fields), or areas of the sky with highpriority targets (e.g., a potentially habitable planet orbiting a Solar-type or dim M-dwarf star).

Given that this small telescope network could keep the ephemerides fresh for transiting exoplanets with large transit depths and bright host stars, it would also be complementary to current transit discovery surveys and efforts, such as CoRoT (Auvergne et al. 2009), HATNet (Bakos et al. 2007, 2013), KELT (Pepper et al. 2003, 2007, 2012), Kepler (Borucki et al. 2010), K2 (Howell et al. 2014), MASCARA (Talens et al. 2017), NGTS (Wheatley et al. 2018), PANOPTES (Guyon et al. 2014), QES (Alsubai et al. 2011), TERMS (Kane et al. 2018), TrES (Alonso et al. 2004), WASP (Pollacco et al. 2006), and XO (McCullough et al. 2005). In addition to ephemerides maintenance, this small telescope network could free up the time for these other surveys to continue their own discovery programs or confirm high-priority targets (e.g., potentially habitable planets around dim M-dwarf stars). The small telescope citizen science network could also join in the efforts of these other surveys to increase the total collection area and world-wide coverage, enabling the pursuit of small and/or dim transits or long-duration transits.

The small telescope network advocated for here would also be complementary to the current on-going TESS Follow-Up Observation Program $^{39}$ (TFOP), which is eliminating TESS candidate false positives and confirming true TESS planetary systems. The TFOP utilizes ground-based time series photometry to eliminate blended eclipsing binaries, spectroscopy to obtain stellar parameters, high resolution imaging to identify potential binary systems, and precision radial velocities to determine the masses of the planets. The TFOP effort will help the community identify some of the best TESS planets for atmospheric characterization from the ground or with space facilities like JWST and ARIEL.

In addition, the small telescope citizen science network would monitor all known transiting exoplanets accessible to them (e.g., large planets with bright host stars; Tables 1-3) to ensure efficient follow-up observations with large platforms, in addition to the other science cases described above. Without continued follow-up, the ephemerides of all the transiting planets will eventually grow stale (see Dragomir et al. 2019a, for a detailed study on TESS targets going stale). The work advocated for here complements the work of the TFOP-so that the mid-transit times will be known sufficiently for efficient future observations. The TFOP is necessary to identify the true planetary systems detected by TESS while a small telescope

\footnotetext{
39 https://heasarc.gsfc.nasa.gov/docs/tess/followup.html
}

network would maintain the quality of the ephemerides to ensure the suitability and efficiency of future characterization observations with JWST, ARIEL, and beyond.

Thus, the ground-based network of smaller $(\leqslant 1 \mathrm{~m})$ telescopes described here would be complementary to current and future ground- and space-based exoplanetary efforts. We find that a network of 166 inch telescopes could measure to $3 \sigma$ the transits of 507 of the 1000 exoplanets expected to be spectroscopically characterized by future missions (e.g., ARIEL) and thus save up to $\sim 20,000$ days of observational overhead. This network would also allow TESS to revisit comparatively less-sampled fields (27.4 days fields) or even new fields (e.g., near the ecliptic). While not impacting the achievement of the prime missions of JWST, ARIEL, or an Astro2020 Decadal, this network would also help ensure the efficient use of these great observatories and potentially enable more science return. This network would also build upon the legacies of Kepler, K2, CoRoT, and other ground-based transit surveys by keeping their ephemerides fresh and extending their measurement baselines to probe for TTVs.

\section{Conclusions}

TESS will revolutionize the field of exoplanet science by providing 10,000+ targets (Barclay et al. 2018) for future transiting exoplanet atmospheric characterization missions (e.g., JWST, ARIEL, and an Astro2020 Decadal Mission, such as HabEx, LUVOIR, or Origins). However, the uncertainty in predicting the time of the next predicted transit for any one of these targets can be significant-larger than the transit duration itself-thereby limiting the efficient use of these large observatories.

We find here that follow-up observations with small $(\leqslant 1 \mathrm{~m})$ telescopes can provide strong constraints on estimating future transits and increase the observational efficiency of these great observatories. We therefore advocate the establishment of a network of small telescopes operated by citizen scientists to provide accurate transit times to the professional astronomer community to help plan upcoming observations. One such proposed network is an open collaboration leveraging small telescopes operated by citizen scientists to provide accurate ephemerides, as described in detail in Zellem et al. $(2019,2020)$, and another is the ExoClock program. ${ }^{40}$

Part of the research was carried out at the Jet Propulsion Laboratory, California Institute of Technology, under contract with the National Aeronautics and Space Administration. Copyright 2020. All rights reserved.

We thank the JPL Exoplanet Science Initiative for partial support of this work.

\footnotetext{
${ }^{40}$ https://www.exoclock.space
} 
This material is based upon work supported by NASA under cooperative agreement award number NNX16AC65A. Any opinions, findings, and conclusions or recommendations expressed in this material are those of the authors and do not necessarily reflect the views of the National Aeronautics and Space Administration.

This research has made use of the NASA Exoplanet Archive, which is operated by the California Institute of Technology, under contract with the National Aeronautics and Space Administration under the Exoplanet Exploration Program.

This research has made use of the SIMBAD database, operated at CDS, Strasbourg, France.

R.T.Z. would like to thank Padi Boyd, Knicole Colon, Sam Halverson, Stella Kafka, Tiffany Kataria, David Latham, and Kevin Stevenson for their helpful discussions.

D. Dragomir acknowledges support provided by NASA through Hubble Fellowship grant HST-HF2-51372.001-A awarded by the Space Telescope Science Institute, which is operated by the Association of Universities for Research in Astronomy, Inc., for NASA, under contract NAS5-26555.

\section{ORCID iDs}

Robert T. Zellem (1) https://orcid.org/0000-0001-7547-0398 Kyle A. Pearson, (1) https://orcid.org/0000-0002-5785-9073 David R. Ciardi (i) https://orcid.org/0000-0002-5741-3047 Franck Marchis, (1) https://orcid.org/0000-0001-7016-7277 Mark R. Swain (i) https://orcid.org/0000-0002-0919-4468 Gael M. Roudier (1) https://orcid.org/0000-0002-7402-7797 Mary Dussault (1) https://orcid.org/0000-0002-8625-6474 Stephen R. Kane iㅣ https://orcid.org/0000-0002-7084-0529 Wilfred Gee (1) https://orcid.org/0000-0002-2931-7605 Lynn Cominsky (10 https://orcid.org/0000-0003-2073-1065 Denise Smith (1) https://orcid.org/0000-0001-6807-5015 Diana Dragomir,, (i) https://orcid.org/0000-0003-2313-467X Brandon Lawton (ii) https://orcid.org/0000-0002-5972-9555 Marc Kuchner (10 https://orcid.org/0000-0002-2387-5489

\section{References}

Agol, E., Cowan, N. B., Knutson, H. A., et al. 2010, ApJ, 721, 1861 Agol, E., Steffen, J., Sari, R., \& Clarkson, W. 2005, MNRAS, 359, 567 Akeson, R. L., Chen, X., Ciardi, D., et al. 2013, PASP, 125, 989 Alonso, R., Auvergne, M., Baglin, A., et al. 2008, A\&A, 482, L21 Alonso, R., Brown, T. M., Torres, G., et al. 2004, ApJL, 613, L153 Alsubai, K. A., Parley, N. R., Bramich, D. M., et al. 2011, MNRAS, 417, 709 Auvergne, M., Bodin, P., Boisnard, L., et al. 2009, A\&A, 506, 411 Bakos, G. Á., Csubry, Z., Penev, K., et al. 2013, PASP, 125, 154 Barclay, T., Pepper, J., \& Quintana, E. V. 2018, ApJS, 239, 2 Barstow, J. K., Aigrain, S., Irwin, P. G. J., Kendrew, S., \& Fletcher, L. N. 2015, MNRAS, 448, 2546

Battersby, C., Armus, L., Bergin, E., et al. 2018, NatAs, 2, 596 Bakos, G. Á, Bayliss, D., Bento, J., et al. 2018, arXiv:1812.09406 Benneke, B., Werner, M., Petigura, E., et al. 2017, ApJ, 834, 187 Bergfors, C., Brandner, W., Daemgen, S., et al. 2013, MNRAS, 428, 182 Berta, Z. K., Charbonneau, D., Bean, J., et al. 2011, ApJ, 736, 12 Bonomo, A. S., Sozzetti, A., Lovis, C., et al. 2014, A\&A, 572, A2 Borucki, W. J., Koch, D., Basri, G., et al. 2010, Sci, 327, 977
Bouma, L. G., Winn, J. N., Kosiarek, J., \& McCullough, P. R. 2017, arXiv: 1705.08891

Braga-Ribas, F., Sicardy, B., Ortiz, J. L., et al. 2014, Natur, 508, 72

Bruno, G., Almenara, J.-M., Barros, S. C. C., et al. 2015, A\&A, 573, A124

Burdanov, A., Benni, P., Sokov, E., et al. 2018, PASP, 130, 074401

Burke, C. J., Bryson, S. T., Mullally, F., et al. 2014, ApJS, 210, 19

Cañas, C. I., Stefansson, G., Monson, A. J., et al. 2019, ApJL, 877, L29

Carter, J. A., Agol, E., Chaplin, W. J., et al. 2012, Sci, 337, 556

Carter, J. A., Winn, J. N., Holman, M. J., et al. 2011, ApJ, 730, 82

Catlin-Groves, C. L. 2012, International Journal of Zoology, 2012, 14

Ciardi, D. R., Beichman, C. A., Horch, E. P., \& Howell, S. B. 2015, ApJ, 805,16

Cochran, W. D., Fabrycky, D. C., Torres, G., et al. 2011, ApJS, 197, 7

Collins, K. A., Collins, K. I., Pepper, J., et al. 2018, AJ, 156, 234

Colón, K. D., Zhou, G., Shporer, A., et al. 2018, AJ, 156, 227

Coughlin, J. L., Mullally, F., Thompson, S. E., et al. 2016, ApJS, 224, 12

Cowan, N. B., Greene, T., Angerhausen, D., et al. 2015, PASP, 127, 311

Croll, B. 2012, JAVSO, 40, 456

Croll, B., Lafreniere, D., Albert, L., et al. 2011, AJ, 141, 30

Crossfield, I. J. M., Barman, T., Hansen, B. M. S., Tanaka, I., \& Kodama, T. 2012, ApJ, 760, 140

Czesla, S., Huber, K. F., Wolter, U., Schröter, S., \& Schmitt, J. H. M. M. 2009, A\&A, 505, 1277

Dai, F., Winn, J. N., Albrecht, S., et al. 2016, ApJ, 823, 115

Dalba, P. A., Kane, S. R., Barclay, T., et al. 2019, PASP, 131, 034401

Dalba, P. A., \& Muirhead, P. S. 2016, ApJL, 826, L7

Damasso, M., Biazzo, K., Bonomo, A. S., et al. 2015, A\&A, 575, A111

Dawson, R. I., Huang, C. X., Lissauer, J. J., et al. 2019, AJ, 158, 65

Delisle, J.-B., Ségransan, D., Dumusque, X., et al. 2018, A\&A, 614, A133

Désert, J.-M., Charbonneau, D., Demory, B.-O., et al. 2011, ApJS, 197, 14

Dragomir, D., Harris, M., Pepper, J., Barclay, T., \& Villanueva, S., Jr. 2019a, ApJ, 875, L7

Dragomir, D., Teske, J., Günther, M. N., et al. 2019b, ApJL, 875, L7

Dumusque, X., Turner, O., Dorn, C., et al. 2019, A\&A, 627, A43

Eastman, J., Gaudi, B. S., \& Agol, E. 2013, PASP, 125, 83

Eaton, J. A., Henry, G. W., \& Fekel, F. C. 2003, in Astrophysics and Space Science Library, ed. T. D. Oswalt, Vol. 288 (Dordrecht: Kluwer Academic Publishers), 189

Edwards, B., Mugnai, L., Tinetti, G., Pascale, E., \& Sarkar, S. 2019, AJ, 157,242

Esposito, M., Armstrong, D. J., Gandolfi, D., et al. 2019, A\&A, 623, A165

Fabrycky, D. C., Ford, E. B., Steffen, J. H., et al. 2012, ApJ, 750, 114

Fischer, D. A., Anglada-Escude, G., Arriagada, P., et al. 2016, PASP, 128, 066001

Ford, E. B. 2005, AJ, 129, 1706

Fossey, S. J., Waldmann, I. P., \& Kipping, D. M. 2009, MNRAS, 396, L16

Fowler, M. J. F. 2019, JBAA, 129, 174

Fraine, J., Deming, D., Benneke, B., et al. 2014, Natur, 513, 526

Franzoni, C., \& Sauermann, H. 2014, Research Policy, 43, 1

Freudenthal, J., von Essen, C., Ofir, A., et al. 2019, A\&A, 628, A108

Gandolfi, D., Barragán, O., Livingston, J. H., et al. 2018, A\&A, 619, L10

Gandolfi, D., Fossati, L., Livingston, J. H., et al. 2019, ApJL, 876, L24

Garcia-Melendo, E., \& McCullough, P. R. 2009, ApJ, 698, 558

Gaudi, B. S., Seager, S., Mennesson, B., et al. 2018, arXiv:1809.09674

Gillon, M., Jehin, E., Lederer, S. M., et al. 2016, Natur, 533, 221

Gillon, M., Triaud, A. H. M. J., Demory, B.-O., et al. 2017, Natur, 542, 456

Goyal, J. M., Mayne, N., Sing, D. K., et al. 2018, MNRAS, 474, 5158

GPy 2012, GPy: A Gaussian Process Framework in Python, http://github. com/SheffieldML/GPy

Günther, M. N., Pozuelos, F. J., Dittmann, J. A., et al. 2019, NatAs, 420, 1099

Guyon, O., Walawender, J., Jovanovic, N., et al. 2014, Proc. SPIE, 9145 , 91453V

Hanuš, J., Viikinkoski, M., Marchis, F., et al. 2017, A\&A, 601, A114

Hellier, C., Anderson, D. R., Gill, S., \& West, R. G. 2019, RNAAS, 3, 156

Henry, G. W. 1999, PASP, 111, 845

Hergenrother, C., \& Hill, D. 2013, MPBu, 40, 164

Hergenrother, C. W., Hill, D. H., Spitz, A., et al. 2014, AAS/DPS Meeting, 46, 213.04

Hergenrother, C. W., Nolan, M. C., Binzel, R. P., et al. 2013, Icar, 226, 663

Holman, M. J., Fabrycky, D. C., Ragozzine, D., et al. 2010, Sci, 330, 51 
Holman, M. J., \& Murray, N. W. 2005, Sci, 307, 1288

Howell, S. B., Sobeck, C., Haas, M., et al. 2014, PASP, 126, 398

Huang, C. X., Shporer, A., Dragomir, D., et al. 2018, arXiv:1807.11129

Huber, D., Chaplin, W. J., Chontos, A., et al. 2019, AJ, 6, 245

Jones, M. I., Brahm, R., Espinoza, N., et al. 2019, A\&A, 625, A16

Jontof-Hutter, D., Lissauer, J. J., Rowe, J. F., \& Fabrycky, D. C. 2014, ApJ, 785,15

Kane, S. R., Mahadevan, S., von Braun, K., Laughlin, G., \& Ciardi, D. R. 2009, PASP, 121, 1386

Kane, S. R., Meshkat, T., \& Turnbull, M. C. 2018, AJ, 156, 267

Kempton, E. M.-R., Bean, J. L., Louie, D. R., et al. 2018, PASP, 130, 114401

Kilpatrick, B. M., Kataria, T., Lewis, N. K., et al. 2019, AJ, 159, 51

Kipping, D., Nesvorný, D., Hartman, J., et al. 2019, MNRAS, 486, 4980

Knutson, H. A., Lewis, N., Fortney, J. J., et al. 2012, ApJ, 754, 22

Kostov, V. B., Schlieder, J. E., Barclay, T., et al. 2019, AJ, 158, 32

Kuchner, M. J., Silverberg, S. M., Bans, A. S., et al. 2016, ApJ, 830, 84

Lauretta, D. S., Balram-Knutson, S. S., Beshore, E., et al. 2017, SSRv, 212,925

Lissauer, J. J., Jontof-Hutter, D., Rowe, J. F., et al. 2013, ApJ, 770, 131

Lissauer, J. J., Ragozzine, D., Fabrycky, D. C., et al. 2011, ApJS, 197, 8

Luque, R., Pallé, E., Kossakowski, D., et al. 2019, A\&A, 628, A39

Mallonn, M., von Essen, C., Herrero, E., et al. 2019, A\&A, 622, A81

Mandel, K., \& Agol, E. 2002, ApJL, 580, L171

Marchis, F., Malvache, A., Marfisi, L., Borot, A., \& Arbouch, E. 2020, AcAau, 166,23

Marshall, P. J., Lintott, C. J., \& Fletcher, L. N. 2015, ARA\&A, 53, 247

Masuda, K. 2014, ApJ, 783, 53

McCullough, P. R., Crouzet, N., Deming, D., \& Madhusudhan, N. 2014, ApJ, 791,55

McCullough, P. R., Stys, J. E., Valenti, J. A., et al. 2005, PASP, 117, 783

Mills, S. M., Fabrycky, D. C., Migaszewski, C., et al. 2016, Natur, 533, 509

Morgan, J., Kerins, E., Awiphan, S., et al. 2019, MNRAS, 486, 796

Mulders, G. D., Mordasini, C., Pascucci, I., et al. 2019, ApJ, 887, 157

Mulders, G. D., Pascucci, I., Apai, D., \& Ciesla, F. J. 2018, AJ, 156, 24

Narita, N., Fukui, A., Ikoma, M., et al. 2013, ApJ, 773, 144

Nesvorný, D., Kipping, D., Terrell, D., et al. 2013, ApJ, 777, 3

Nesvorný, D., \& Morbidelli, A. 2008, ApJ, 688, 636

Newton, E. R., Mann, A. W., Tofflemire, B. M., et al. 2019, ApJL, 880, L17

Nielsen, L. D., Bouchy, F., Turner, O., et al. 2019, A\&A, 623, A100

Bakos, G. Á, Noyes, R. W., Kovács, G., et al. 2007, ApJ, 656, 552

Oshagh, M., Santos, N. C., Ehrenreich, D., et al. 2014, A\&A, 568, A99

Palle, E., Nowak, G., Luque, R., et al. 2018, A\&A, 623, A41

Pearson, K. A. 2019, AJ, 158, 243

Penn, M. J., Baer, R., Bosh, R., et al. 2017, PASP, 129, 015005

Penn, M. J., Baer, R., Walter, D., et al. 2019, PASP, 132, 014201

Pepper, J., Gould, A., \& Depoy, D. L. 2003, AcA, 53, 213

Pepper, J., Kuhn, R. B., Siverd, R., James, D., \& Stassun, K. 2012, PASP, 124,230

Pepper, J., Pogge, R. W., DePoy, D. L., et al. 2007, PASP, 119, 923

Petigura, E. A., Benneke, B., Batygin, K., et al. 2018, AJ, 156, 89

Petigura, E. A., Howard, A. W., Lopez, E. D., et al. 2016, ApJ, 818, 36

Petigura, E. A., Marcy, G. W., \& Howard, A. W. 2013, ApJ, 770, 69
Petigura, E. A., Sinukoff, E., Lopez, E. D., et al. 2017, AJ, 153, 142 Poddaný, S., Brát, L., \& Pejcha, O. 2010, NewA, 15, 297

Pollacco, D. L., Skillen, I., \& Collier Cameron, A. 2006, PASP, 118, 1407

Pont, F., Knutson, H., Gilliland, R. L., Moutou, C., \& Charbonneau, D. 2008, MNRAS, 385, 109

Pravec, P., Scheirich, P., Kušnirák, P., et al. 2016, Icar, 267, 267

Quinn, S. N., Becker, J. C., Rodriguez, J. E., et al. 2019, AJ, 158, 177

Rackham, B., Espinoza, N., Apai, D., et al. 2017, ApJ, 834, 151

Rackham, B. V., Apai, D., \& Giampapa, M. S. 2018, ApJ, 853, 122

Ricker, G. R., Winn, J. N., Vanderspek, R., et al. 2014, Proc. SPIE, 9143, 914320

Rodriguez, J. E., Becker, J. C., Eastman, J. D., et al. 2018, AJ, 156, 245

Rodriguez, J. E., Quinn, S. N., Huang, C. X., et al. 2019, AJ, 157, 191

Rowe, J. F., Bryson, S. T., Marcy, G. W., et al. 2014, ApJ, 784, 45

Rowe, J. F., Coughlin, J. L., Antoci, V., et al. 2015, ApJS, 217, 16

Sadler, P. M., Gould, R. R., Leiker, P. S., et al. 2001, JSEdT, 10, 39

Sanchis-Ojeda, R., Fabrycky, D. C., Winn, J. N., et al. 2012, Natur, 487, 449

Silva-Valio, A. 2008, ApJL, 683, L179

Sing, D. K., Pont, F., Aigrain, S., et al. 2011, MNRAS, 416, 1443

Steffen, J. H., Fabrycky, D. C., Agol, E., et al. 2013, MNRAS, 428, 1077

Stevenson, K. B., Bean, J. L., \& Seifahrt, A. 2014, AJ, 147, 161

Sullivan, P. W., Winn, J. N., Berta-Thompson, Z. K., et al. 2015, ApJ, 809, 77

Swain, M. R., Deroo, P., Griffith, C. A., et al. 2010, Natur, 463, 637

Talens, G. J. J., Spronck, J. F. P., Lesage, A.-L., et al. 2017, A\&A, 601, A11

The LUVOIR Team 2018, arXiv:1809.09668

Timerson, B., Brooks, J., Conard, S., et al. 2013, P\&SS, 87, 78

Tinetti, G., Drossart, P., Eccleston, P., et al. 2016, Proc. SPIE, 9904, 99041X

Tinetti, G., Drossart, P., Eccleston, P., et al. 2018, ExA, 46, 135

Trifonov, T., Rybizki, J., \& Kürster, M. 2019, A\&A, 622, L7

Vanderburg, A., Huang, C. X., Rodriguez, J. E., et al. 2019, ApJL, 881, L19

Vanderspek, R., Huang, C. X., Vanderburg, A., et al. 2019, ApJL, 871, L24

Virtanen, P., Gommers, R., Oliphant, T. E., et al. 2019, arXiv:1907.10121

von Essen, C., Ofir, A., Dreizler, S., et al. 2018, A\&A, 615, A79

Wang, S., Jones, M., Shporer, A., et al. 2019, AJ, 157, 51

Wang, Y.-H., Wang, S., Hinse, T. C., et al. 2019b, AJ, 157, 82

Watson, D., \& Floridi, L. 2018, Synthese, 195, 741

Weiss, L. M., Marcy, G. W., Rowe, J. F., et al. 2013, ApJ, 768, 14

Wenger, M., Ochsenbein, F., Egret, D., et al. 2000, A\&AS, 143, 9

Wheatley, P. J., West, R. G., Goad, M. R., et al. 2018, MNRAS, 475, 4476

Wiggins, A., \& Crowston, K. 2011, in 2011 44th Hawaii Int. Conf. on System Sciences (Piscataway, NJ: IEEE)

Winters, J. G., Medina, A. A., Irwin, J. M., et al. 2019, arXiv:1906.10147

Wolter, U., Schmitt, J. H. M. M., Huber, K. F., et al. 2009, A\&A, 504, 561

Xie, J.-W. 2013, ApJS, 208, 22

Zellem, R. T., Biferno, A., Ciardi, D. R., et al. 2019, BAAS, 51, 416

Zellem, R. T., Griffith, C. A., Pearson, K. A., et al. 2015, ApJ, 810, 11

Zellem, R. T., Pearson, K. A., Ciardi, D., et al. 2020, AAS Meeting, 52, 337.07

Zellem, R. T., Swain, M. R., Cowan, N. B., et al. 2019b, PASP, 131, 094401

Zellem, R. T., Swain, M. R., Roudier, G., et al. 2017, ApJ, 844, 27

Zhang, Z., Zhou, Y., Rackham, B. V., \& Apai, D. 2018, AJ, 156, 178 